\title{
A Variational Approach to Contracting under Imperfect Observations*
}

\author{
Agostino Capponi \\ School of Industrial Engineering \\ Purdue University, West Lafayette, IN 47907 \\ Email: capponi@purdue.edu \\ Jakša Cvitanić \\ Division of the Humanities and Social Sciences \\ California Institute of Technology, Pasadena, CA 91125 \\ Email: cvitanic@hss.caltech.edu \\ Türkay Yolcu \\ Department of Mathematics \\ Purdue University, West Lafayette, IN 47907 \\ Email: tyolcu@math.purdue.edu
}

December 14, 2011

\footnotetext{
*The research of J. Cvitanić was supported in part by NSF grant 10-08219.
} 


\begin{abstract}
We consider a continuous time model of the project value process that can only be observed with noise, and we allow for the possibility that the manager in charge of the project can misrepresent the observed value. The manager is compensated by the shareholders, based on the filtering estimate of the project outcome. By means of a variational calculus methodology, novel for this kind of problems, we are able to compute in closed form the optimal pay-per-performance sensitivity of the compensation and the optimal misreporting action. We illustrate our theoretical predictions through a detailed comparative statics analysis, which indicates that the shareholders induce the manager to increase the amount of misreporting over time.
\end{abstract}

Keywords: variational calculus; stochastic filtering; optimal compensation; hidden action; risksharing.

\title{
1 Introduction
}

We consider a continuous-time model of the firm's value process in which shareholders hire a manager to run the firm, and pay him a part of the proceeds as compensation. The payment is decided by a contract between the two parties. The first and seminal continuous-time model was developed in Holmstrom and Milgrom (1987), and considers the case of hidden action, in which the manager's control (effort) of the drift of the project value process cannot be contracted upon, either because it is unobserved by the shareholders, and/or because that is not legally enforceable. Their work was generalized and extended by many authors, including Schättler and Sung (1993, 1997). See also Ou-Yang (2005), Ju and Wan (2010), and Cuoco and Kaniel (2011). Cvitanić, Wang and Zhang (2009) uses the stochastic maximum principle and forward-backward stochastic differential Equations (FBSDEs) to characterize the optimal compensation for more general utility functions. Giat et al. (2010) consider a model in which the project value is observed, but its risk premium (drift term) is not observed, and the shareholders and the manager may have different prior beliefs about it. Goukasian and Wan (2010) consider a hidden action framework with multiple agents who only care about their relative position and study the impact of this assumption on the optimal contract.

The distinguishing features of our model are: (i) the actual project output is not observed directly, but only with noise; (ii) the manager can bias the observations of the actual project output by his actions. In justifying the modeling assumption (i), let us mention that the presence of accounting noise in reports has been well documented in the literature, and has been found to be an important determinant of corporate credit spreads, see, e.g., Capponi and Cvitanić (2008). Assumption (ii) means that the manager can misreport the accounting reports in order to increase his own utility, albeit at a cost. Even if the misreporting action is hidden, the shareholders are aware of its possibility, and can correctly compute the amount of the manager's misreporting in equilibrium. Moreover, the manager incurs a penalty for applying misreporting action, which may be interpreted as a reduced form model of the reputation concerns, for example from negative effects on him of potential auditing. Higher auditing frequency would correspond to increasing the cost of the misreporting action.

We assume that the shareholders (like the manager) have short-term objectives, and are not concerned about the long-term value of the firm. Further, we assume that the outside market is unable to detect, or correctly estimate the level of misreporting in the short run. This can be due to several reasons. For example, outside investors may lack information on the utility functions of the manager and/or the shareholders, or on which compensation contract is promised to the manager. Alternatively, we can think of a part of the market as composed of unsophisticated investors, for example small retail investors, who are more likely to trust the released reports, and would take 
longer time to question their validity due to their inability to well understand firms' disclosures, see Fishman and Hagerty (2003). Clearly, misreporting could be detected or estimated in the long run, effectively bursting a "bubble" created by it. We assume this can happen only after the contract is paid, and thus it is not modeled here ${ }^{1}$.

As misreporting affects the probability distribution of the observed output process but is not correctly accounted for by the market, it results in the market producing biased estimates of the output process. This implies that both shareholders and managers could extract benefits from misreporting, as they would be able to sell the firm to the market in the short term at a premium over the actual value.

We are interested in characterizing the behavior of the optimal pay-per-performance sensitivity and misreporting action applied by the manager over time under a hidden action model. In order to test the robustness of our qualitative conclusions, we also consider the risk sharing informational framework, in which the misreporting action is decided by shareholders and manager together, so as to maximize their joint utility. We assume that compensation is linear in the market estimate of the value of the output process, and that both manager and shareholders have exponential utilities. The partial observability of the output makes the optimization problem non-standard, thus requiring a proper reformulation as a free-boundary calculus of variation problem with constraints. This differs from previous works in the literature, where full information about the output is available. To this end, we apply a novel variational methodology based on time dependent Lagrangian multipliers and we are able to obtain an explicit solution to the problem via the Euler-Lagrange equation, thus facilitate a comparative statics analysis. Despite the methodology is specialized to the above described misreporting problem, the variational techniques developed in this paper can be viable to analyze moral hazard problems where multiple non-contractible actions are taken.

Different methodologies using variational analysis have been applied to principal agent theory within an adverse selection framework. Those include Carlier, Ekeland and Touzi (2007), who show existence and uniqueness of the optimal derivative design, and Figalli, Lom and McCann (2011) who derive uniqueness and stability of the principal's optimum strategy. Horst and MorenoBromberg (2008) generalize the principal's utility function by assuming that contract design exposes the principal to additional risk, measured by a convex risk measure.

Through our analysis, we find that the shareholders decrease the pay-per-performance sensitivity offered to the manager when the level of observation noise increases, which in turns leads the manager to apply a smaller amount of misreporting. This happens because, as the intensity of the noise gets higher, the gain resulting from the fact that the market is unaware of misreporting becomes smaller and smaller. Moreover, we find that it is optimal for shareholders to induce the manager to increase the level of misreporting over time. Thus, if regulators were to undertake early auditing actions, they would prevent higher and higher levels of accounting irregularities from occurring.

The remainder of the paper is organized as follows. Section 2 describes the model. Section 3 introduces and analyzes the problem with hidden misreporting action in detail. Section 4 deals with the risk-sharing problem of maximizing the joint welfare. Section 5 performs a comparative statics analysis. Section 6 concludes our study. Longer proofs are delegated to Section 7.

\footnotetext{
${ }^{1}$ More direct and mathematically sophisticated modeling of price bubbles is an active area in research; see, e.g., Loewenstein and Willard (2000), Cox and Hobson (2005), Jarrow, Protter, and Shimbo (2010).
} 


\section{The Model}

We work on a probability space $\left(\Omega, \mathcal{F}_{T}, \mathbb{P}\right)$, where $T$ is a fixed time horizon. We assume that the project value (profit/loss, firm value, outcome) process is given by

$$
d x_{t}=\theta \epsilon d t+\epsilon d W_{t}
$$

for some constants $\theta>0, \epsilon>0$, and a $\mathcal{F}_{t}$-Brownian motion $W_{t}$. We assume that $x_{0}$ is observed, for simplicity. The project value has to be shared between the owners (the shareholders) and the manager. We assume that the value process is not directly observed, instead, what is observed is process $y$ given by

$$
d y_{t}=x_{t} d t+\sigma d Z_{t}^{0}
$$

where $Z_{t}^{0}$ is a $\mathcal{F}_{t}$-Brownian motion independent of $W_{t}$. We denote by $\mathcal{F}_{t}^{y}$ the filtration generated by the observation process $\left\{y_{t}\right\}$. Here, $\sigma>0$ models the accounting noise. Given a deterministic bounded function $a_{t}{ }^{2}$ defined on the interval $[0, T]$, which represents the manager's action, we define

$$
\begin{aligned}
Z_{t}^{a} & =Z_{t}^{0}-\int_{0}^{t} \frac{a_{s}}{\sigma} d s \\
M_{t}^{a} & =\exp \left\{\int_{0}^{t} \frac{a_{s}}{\sigma} d Z_{s}^{0}-\frac{1}{2} \int_{0}^{t}\left(\frac{a_{s}}{\sigma}\right)^{2} d s\right\} \\
\mathbb{P}^{a}(A) & =\mathbb{E}\left[M_{T}^{a} \mathbf{1}_{A}\right]
\end{aligned}
$$

Then, we know by Girsanov theorem that $\mathbb{P}^{a}(A)$ is a probability measure and $Z_{t}^{a}$ is a $\left(\mathcal{F}_{t}, \mathbb{P}^{a}\right)$ Brownian motion. The manager's choice of $a_{t}$ corresponds to the choice of the probability measure $\mathbb{P}^{a}$, and thus to the choice of the distribution of the observation process $y_{t}$. In other words, the manager adds the misreporting amount $a_{t}$ to the rate of the actual outcome process $x_{t}$, thus (possibly) introducing bias.

For a given function $a_{t}$, we introduce the filter process,

$$
\hat{x}_{t}^{a}=\mathbb{E}_{t}^{a}\left[x_{t}\right] \triangleq \mathbb{E}^{\mathbb{P}^{a}}\left[x_{t} \mid \mathcal{F}_{t}^{y}\right]
$$

Remark 2.1. Our setup models misreporting via a change of measure method, which is a standard modeling procedure for hidden action problems, including the original Holmstrom-Milgrom model. One novelty here is that we will use not only quantities computed under measure $\mathbb{P}^{a}$, but, as we shall see, also $\hat{x}^{0}$, the estimate of the output under the (incorrect) assumption that there is no misreporting.

We can write

$$
d y_{t}=\left[\hat{x}_{t}^{a}+a_{t}\right] d t+\sigma d \hat{Z}_{t}^{a}
$$

where

$$
\hat{Z}_{t}^{a}=Z_{t}^{a}+\frac{1}{\sigma} \int_{0}^{t}\left[x_{s}-\hat{x}_{s}^{a}\right] d s
$$

is the innovation process for our filter, which is well known to be a $\left(\mathcal{F}_{t}^{y}, \mathbb{P}^{a}\right)$-Brownian motion, see

\footnotetext{
${ }^{2}$ We assume deterministic action, and, later below, linear contracts, for tractability reasons. In the classical Holmstrom-Milgrom model, the optimal action is, in fact, deterministic and the optimal contract is linear. It is possible that also in our model these assumptions are without loss of generality, but we don't have a proof.
} 
Liptser and Shiryaev (2000). Note that the classical Kalman-Bucy filter gives

$$
d \hat{x}_{t}^{a}=\theta \epsilon d t+\frac{v_{t}}{\sigma} d \hat{Z}_{t}^{a}
$$

where $v_{t}$ satisfy the first order differential equation

$$
v_{t}^{\prime}=\epsilon^{2}-\frac{1}{\sigma^{2}} v_{t}^{2}
$$

with solution

$$
v_{t}=\epsilon \sigma \frac{e^{\frac{\epsilon t}{\sigma}}-e^{-\frac{\epsilon t}{\sigma}}}{e^{\frac{\epsilon t}{\sigma}}+e^{-\frac{\epsilon t}{\sigma}}}=\epsilon \sigma \tanh \left(\frac{\epsilon t}{\sigma}\right)
$$

We have the following useful relations, which will be extensively used later:

Lemma 2.1. We have

$$
\hat{Z}_{t}^{0}=\int_{0}^{t} \frac{\sigma}{v_{s}} d \hat{x}_{s}^{0}-\int_{0}^{t} \frac{\epsilon \sigma \theta}{v_{s}} d s
$$

and so, $\hat{Z}_{t}^{a}$ is expressed in terms of $\hat{x}_{t}^{0}$ as

$$
\hat{Z}_{t}^{a}=\hat{Z}_{t}^{0}-\int_{0}^{t} \frac{a_{s}}{\sigma} d s+\int_{0}^{t} \frac{\hat{x}_{s}^{0}-\hat{x}_{s}^{a}}{\sigma} d s .
$$

Moreover, $\hat{x}_{t}^{0}$ may be expressed in terms of $\hat{Z}_{t}^{a}$ as

$$
d \hat{x}_{t}^{0}=\epsilon \theta d t+\frac{v_{t}}{\sigma} d \hat{Z}_{t}^{a}+\frac{v_{t} a_{t}}{\sigma^{2}} d t-\frac{v_{t}}{\sigma^{2}}\left(\hat{x}_{t}^{0}-\hat{x}_{t}^{a}\right) d t
$$

The proof of Lemma 2.1 is provided in Section 7.1. If we assume that $a_{t}$ is deterministic, as we will, the filter mismatch term $\hat{x}_{t}^{0}-\hat{x}_{t}^{a}$ may be explicitly computed. The result is given in the following lemma, whose proof is presented in Section 7.1.

\section{Lemma 2.2.}

$$
\hat{x}_{t}^{0}-\hat{x}_{t}^{a}=\frac{\epsilon \int_{0}^{t} \sinh \left(\frac{\epsilon s}{\sigma}\right) a_{s} d s}{\sigma \cosh \left(\frac{\epsilon t}{\sigma}\right)} .
$$

We restrict our analysis to the linear contracts of the form

$$
C_{T}=c+\int_{0}^{T} \alpha_{t} d \hat{x}_{t}^{0}
$$

where $\alpha_{t}$ is deterministic. The payment $C_{T}$ is thus an $\mathcal{F}_{T}^{y}$-measurable random variable, and should be interpreted as a payment in cash, the amount of which depends on random outcomes of $\hat{x}^{0}$ by time $T$. The justification for using $\hat{x}^{0}$ rather than $\hat{x}^{a}$ is that the payments legally have to be offered under the assumption that there is no misreporting. In what follows, we make the following assumption

Assumption 2.1. Both the misreporting action $a_{t}$ and the pay-per-performance sensitivity (PPS) $\alpha_{t}$ belong to the set of deterministic continuously differentiable functions on the interval $[0, T]$.

We will use $\alpha_{t}^{H A}$ and $a_{t}^{H A}$ to denote, respectively, the optimal pay-per-performance sensitivity, and optimal misreporting action in this hidden action framework. We, instead, use $\alpha_{t}^{R S}$ and $a_{t}^{R S}$ later below, to denote, respectively, the optimal pay-per-performance sensitivity, and optimal misreporting action in the risk-sharing framework. 


\section{Hidden Action}

We assume that the manager is maximizing, over the choice of $a_{t}$, the expected utility

$$
\mathbb{E}^{a}\left[U_{1}\left(C_{T}-G_{T}\right)\right] \triangleq \mathbb{E}^{\mathbb{P}^{a}}\left[U_{1}\left(C_{T}-G_{T}\right)\right]
$$

where $\mathbb{E}^{a}$ is the expectation corresponding to the action process $a, C_{T}$ is the payment from the shareholders to the manager at time $T$, and $G_{T}$ is the cost the manager faces. We assume the latter to be of the form

$$
G_{T}=\int_{0}^{T} g\left(a_{t}\right) d t
$$

where $g(x)$ is assumed to be a strictly convex function so that

$$
g(x) \geq \xi \frac{x^{2}}{2}
$$

As already mentioned in the introduction, this cost may be thought of as a reduced form modeling of the negative reputation effects on the manager if his misreporting is discovered by the market. The information structure in our model is as follows.

- The manager observes process $y$, and chooses action $a$.

- The shareholders observe process $y$, and do not necessarily observe action $a$, but they compute it correctly from knowing everything about the model. Therefore, they can also produce an unbiased estimate of the outcome process using the observations.

- The market (outside investors) can not compute exactly the misreporting level optimal for the manager, due to lack of information.

For simplicity, we will assume that the outside investors estimate the output process by $\hat{x}_{t}^{0}$, that is, assuming no misreporting. This is approximately true if their prior for action $a$ is that of no misreporting $\left(a_{t} \equiv 0\right)$, and the time horizon $T$ is short. Our results below would remain qualitatively the same if we assumed that the market value of the firm at time $T$ is given by $\hat{x}^{a_{f}}$ for some fixed "conjectured" level of misreporting $a_{f}$, different from the actual level. Such an assumption is justified if the market does not know exactly the manager's or the shareholders' utility functions and/or the cost function, or does not know other parameters of the model, but tries to form inferences about the misreporting level over time through observations of the output process. That would also be the case if with some positive probability $p$ the market uses $\hat{x}^{0}$ as the estimate, and with probability $1-p$ it uses the unbiased estimate $\hat{x}^{a}$. We focus on the setting in which they use $\hat{x}^{0}$, to examine the effects of misreporting in this extreme case.

Let us also mention that, even though the true value of the firm may be known eventually (or asymptotically) as time goes by, so that the long-term shareholders will be awarded the actual value, short-term shareholders that we are modeling are hoping to be able to sell off their shares at the value inflated by misreporting. Effectively, as already stated in the introduction, there is a "bubble" that we assume may burst only after time $T$, and is thus not modeled here.

The shareholders' problem is to maximize over $C_{T}$ the expected value

$$
\mathbb{E}^{a}\left[U_{2}\left(\hat{x}_{T}^{0}-C_{T}\right)\right]
$$


under the constraint that the manager's expected utility is at least $R_{0}$, for some given constant $R_{0}$. The quantity $\hat{x}_{T}^{0}-C_{T}$ can be interpreted as the residual gain for the shareholders, i.e. the proceeds from the sale of the firm to the market, after paying the manager. Moreover, the fact that the shareholders take expectation under the measure $\mathbb{P}^{a}$ corresponds to the assumption that they can compute the level of the manager's misreporting exactly.

In what follows, we make the following assumption

Assumption 3.2. $\alpha_{t} \geq 0$.

This is a natural condition, guaranteeing that the compensation is higher with higher returns in output. We restrict our attention to exponential utilities, assuming that

$$
U_{1}(x)=-e^{-\gamma_{1} x} \quad U_{2}(x)=-e^{-\gamma_{2} x}
$$

We now introduce the following quantities

$$
p_{t} \triangleq \int_{0}^{t} \sinh \left(\frac{\epsilon s}{\sigma}\right) a_{s} d s
$$

and

$$
q_{t} \triangleq \int_{t}^{T} g^{\prime}\left(a_{s}\right) d s
$$

\subsection{Optimal misreporting given contract}

In this subsection we study the optimal misreporting action for a given contract. We establish a relation which connects given $\alpha_{t}$ to the optimal $a_{t}$. Before giving the main result, we give an auxiliary lemma.

Lemma 3.3. If $a_{t}, t>0$, minimizes the functional

$$
\int_{t}^{T}\left[\frac{1}{2} \gamma_{1} \alpha_{s}^{2} \frac{v_{s}^{2}}{\sigma^{2}}-\theta \epsilon \alpha_{s}+g\left(a_{s}\right)-\alpha_{s} \frac{v_{s}}{\sigma^{2}}\left(a_{s}-\left(\frac{\epsilon \int_{0}^{s} \sinh \left(\frac{\epsilon r}{\sigma}\right) a_{r} d r}{\sigma \cosh \left(\frac{\epsilon s}{\sigma}\right)}\right)\right)\right] d s
$$

then the following relation must hold for $t>0$

$$
\alpha_{t}=\frac{\sigma^{2}}{v_{t}} g^{\prime}\left(a_{t}\right)+\int_{t}^{T} g^{\prime}\left(a_{s}\right) d s .
$$

The proof of the lemma is presented in Section 7.2. We then have the following result which establishes the relationship between the optimal pay-per-performance sensitivity and misreporting action.

Proposition 3.1. The pay-per-performance sensitivity $\alpha_{t}^{H A}$ corresponding to the manager's optimal misreporting action $a_{t}^{H A}$ satisfies

$$
\alpha_{t}^{H A}=\frac{\sigma^{2}}{v_{t}} g^{\prime}\left(a_{t}^{H A}\right)+\int_{t}^{T} g^{\prime}\left(a_{s}^{H A}\right) d s .
$$


Proof. The manager maximizes over $a_{t}$ his utility function

$$
\begin{aligned}
\mathbb{E}^{a}\left[U_{1}\left(C_{T}-G_{T}\right)\right] & =\mathbb{E}^{a}\left[-\exp \left\{-\gamma_{1}\left(C_{T}-\int_{0}^{T} g\left(a_{t}\right) d t\right)\right\}\right] \\
& =\mathbb{E}^{a}\left[-\exp \left\{-\gamma_{1}\left(c+\int_{0}^{T} \alpha_{t} d \hat{x}_{t}^{0}-\int_{0}^{T} g\left(a_{t}\right)\right) d t\right\}\right] \\
& =-\exp \{\Sigma\}
\end{aligned}
$$

where

$$
\Sigma=-\gamma_{1}\left(c+\int_{0}^{T} \alpha_{t}\left(\theta \epsilon-\frac{1}{2} \alpha_{t} \gamma_{1} \frac{v_{t}^{2}}{\sigma^{2}}+\frac{v_{t} a_{t}}{\sigma^{2}}-\frac{v_{t}}{\sigma^{2}}\left(\frac{\epsilon \int_{0}^{t} \sinh \left(\frac{\epsilon s}{\sigma}\right) a_{s} d s}{\sigma \cosh \left(\frac{\epsilon t}{\sigma}\right)}\right)\right) d t\right)+\gamma_{1} \int_{0}^{T} g\left(a_{t}\right) d t
$$

Note that the last equality (3.8) follows from using the expression for $\hat{x}_{t}^{0}$ given in Eq. (2.12), and the fact that, for a given deterministic function $\psi(t)$, we have

$$
\mathbb{E}^{a}\left[\exp \left(\int_{0}^{T} \psi(t) d \hat{Z}_{t}^{a}\right)\right]=\exp \left(\frac{1}{2} \int_{0}^{T} \psi(t)^{2} d t\right)
$$

Maximizing the above expectation is equivalent to the problem

$$
a_{t}^{H A} \triangleq \operatorname{argmin}_{a} \int_{0}^{T}\left[\frac{1}{2} \gamma_{1} \alpha_{t}^{2} \frac{v_{t}^{2}}{\sigma^{2}}-\theta \epsilon \alpha_{t}+g\left(a_{t}\right)-\alpha_{t} \frac{v_{t}}{\sigma^{2}}\left(a_{t}-\left(\frac{\epsilon \int_{0}^{t} \sinh \left(\frac{\epsilon s}{\sigma}\right) a_{s} d s}{\sigma \cosh \left(\frac{\epsilon t}{\sigma}\right)}\right)\right)\right] d t .
$$

Application of Lemma 3.3 thus yields the result.

\subsection{Optimal Contract}

The shareholders want to maximize, over $\alpha_{t}$, the expected utility given by Eq. (3.2), under the reservation constraint that the manager expected utility, given by Eq. (3.8), is equal to $R_{0}$. From this constraint, we obtain

$$
\begin{aligned}
R_{0} & =-\mathbb{E}\left[\exp \left\{-\gamma_{1} c-\gamma_{1} \int_{0}^{T} \alpha_{t} d \hat{x}_{t}^{0}+\gamma_{1} g\left(a_{t}\right) d t\right\}\right] \\
& =-\exp \left\{-\gamma_{1}\left[c+\int_{0}^{T}\left(\alpha_{t}\left(\epsilon \theta-\frac{\gamma_{1}}{2} \frac{v_{t}^{2} \alpha_{t}}{\sigma^{2}}+\frac{v_{t} a_{t}}{\sigma^{2}}-\frac{v_{t}}{\sigma^{2}}\left(\hat{x}_{t}^{0}-\hat{x}_{t}^{a}\right)\right)-g\left(a_{t}\right)\right) d t\right]\right\}
\end{aligned}
$$

leading to

$$
e^{-\gamma_{1} c}=-R_{0} \exp \left\{\gamma_{1}\left[\int_{0}^{T}\left(\alpha_{t}\left(\epsilon \theta-\frac{\gamma_{1}}{2} \frac{v_{t}^{2} \alpha_{t}}{\sigma^{2}}+\frac{v_{t} a_{t}}{\sigma^{2}}-\frac{v_{t}}{\sigma^{2}}\left(\hat{x}_{t}^{0}-\hat{x}_{t}^{a}\right)\right)-g\left(a_{t}\right)\right) d t\right]\right\}
$$

and therefore, solving for $c$ in the above equation, we arrive at

$$
c=-\frac{1}{\gamma_{1}} \log \left(-R_{0}\right)-\int_{0}^{T}\left(\alpha_{t}\left[\theta \epsilon-\frac{1}{2} \frac{v_{t}^{2}}{\sigma^{2}} \gamma_{1} \alpha_{t}+\frac{v_{t} a_{t}}{\sigma^{2}}-\frac{v_{t}}{\sigma}\left(\hat{x}_{t}^{0}-\hat{x}_{t}^{a}\right)\right]-g\left(a_{t}\right)\right) d t .
$$


Using the expression for $c$ given in Eq. (3.11), and using Eq. (2.12) to express everything in terms of $\hat{Z}_{t}^{a}$, we can immediately verify that

$$
\begin{aligned}
C_{T} & =c+\int_{0}^{T} \alpha_{t} d \hat{x}_{t}^{0} \\
& =-\frac{1}{\gamma_{1}} \log \left(-R_{0}\right)+\frac{\gamma_{1}}{2} \int_{0}^{T} \alpha_{t}^{2} \frac{v_{t}^{2}}{\sigma^{2}} d t+\int_{0}^{T} \alpha_{t} \frac{v_{t}}{\sigma} d \hat{Z}_{t}^{a}+\int_{0}^{T} g\left(a_{t}\right) d t .
\end{aligned}
$$

Plugging the expression for $\hat{x}_{T}^{0}$ obtain from integrating the SDE in Eq. (2.12) and the expression for $C_{T}$ given by Eq. (3.12) into Eq. (3.2), where we recall that $U_{2}(x)=-e^{-\gamma_{2} x}$, we get that we need to minimize, over $a_{t}$,

$$
\begin{aligned}
\mathbb{E}^{a}\left[\exp \left\{-\gamma_{2}\left(\hat{x}_{T}^{0}-C_{T}\right)\right\}\right]= & \mathbb{E}^{a}\left[\operatorname { e x p } \left\{-\gamma_{2}\left[x_{0}+\int_{0}^{T} \epsilon \theta d t\right]+\frac{\gamma_{2} \gamma_{1}}{2} \int_{0}^{T} \frac{\alpha_{t}^{2} v_{t}^{2}}{\sigma^{2}} d t\right.\right. \\
& +\gamma_{2} \int_{0}^{T}\left[g\left(a_{t}\right)-\frac{a_{t} v_{t}}{\sigma^{2}}\right] d t-\gamma_{2} \int_{0}^{T} \frac{v_{t}}{\sigma} d \hat{Z}_{t}^{a} \\
& \left.\left.+\gamma_{2} \int_{0}^{T} \alpha_{t} \frac{v_{t}}{\sigma} d \hat{Z}_{t}^{a}+\gamma_{2} \int_{0}^{T} \frac{v_{t}}{\sigma} \frac{\epsilon \int_{0}^{t} \sinh \left(\frac{\epsilon s}{\sigma}\right) a_{s} d s}{\sigma^{2} \cosh \left(\frac{\epsilon t}{\sigma}\right)} d t\right\}\right] .
\end{aligned}
$$

As $a_{t}$ is deterministic, for a given square-integrable deterministic function $\psi$, the expectation above can be computed by using Eq. (3.9). Therefore, we want to minimize

$$
A\left(t, a_{t}\right) \triangleq \int_{0}^{T}\left[\frac{\gamma_{1}}{2} \frac{\alpha_{t}^{2} v_{t}^{2}}{\sigma^{2}}+g\left(a_{t}\right)-\frac{1}{\sigma^{2}} a_{t} v_{t}+\frac{\gamma_{2}}{2}\left(-\frac{v_{t}}{\sigma}+\alpha_{t} \frac{v_{t}}{\sigma}\right)^{2}+\frac{v_{t}}{\sigma} \frac{\epsilon \int_{0}^{t} \sinh \left(\frac{\epsilon s}{\sigma}\right) a_{s} d s}{\sigma^{2} \cosh \left(\frac{\epsilon t}{\sigma}\right)}\right] d t
$$

where the expression for $\alpha_{t}$ is given in Eq. (3.7). It follows from Eq. (3.4) and Eq. (3.5) that

$$
p_{0}=0, \quad q_{T}=0,
$$

and for $t>0$ we have

$$
p_{t}^{\prime}=\sinh \left(\frac{\epsilon t}{\sigma}\right) a_{t}, \quad q_{t}^{\prime}=-g^{\prime}\left(a_{t}\right)
$$

from which we obtain

$$
q_{t}^{\prime}=-g^{\prime}\left(\operatorname{csch}\left(\frac{\epsilon t}{\sigma}\right) p_{t}^{\prime}\right), \quad(t>0)
$$

Observe that Eq. (3.4) and Eq. (3.5) turn the integral in Eq. (3.14) into

$$
A^{L}\left(t, p_{t}, q_{t}\right) \triangleq \int_{0}^{T} L\left(t, p_{t}, q_{t}, p_{t}^{\prime}, q_{t}^{\prime}\right) d t
$$


where the integrand is given by

$$
\begin{aligned}
L\left(t, p_{t}, q_{t}, p_{t}^{\prime}, q_{t}^{\prime}\right) \triangleq & \frac{\gamma_{1}}{2}\left[\sigma q_{t}^{\prime}-\epsilon \tanh \left(\frac{\epsilon t}{\sigma}\right) q_{t}\right]^{2} \\
& +\frac{1}{2} \xi\left(\operatorname{csch}\left(\frac{\epsilon t}{\sigma}\right) p_{t}^{\prime}\right)^{2}-\frac{\epsilon}{\sigma} \operatorname{sech}\left(\frac{\epsilon t}{\sigma}\right) p_{t}^{\prime} \\
& +\frac{\gamma_{2}}{2}\left[\sigma q_{t}^{\prime}+\epsilon \tanh \left(\frac{\epsilon t}{\sigma}\right)\left(1-q_{t}\right)\right]^{2} \\
& +\frac{\epsilon^{2}}{\sigma^{2}} \tanh \left(\frac{\epsilon t}{\sigma}\right) \operatorname{sech}\left(\frac{\epsilon t}{\sigma}\right) p_{t} .
\end{aligned}
$$

Before stating the main result, we give a few auxiliary lemmas.

Lemma 3.4. There exist unique optimal $a_{t}, p_{t}$, and $q_{t}$ such that

$$
\min _{a_{t}}\left\{A\left(t, a_{t}\right): a_{t} \text { satisfies }(3.6)\right\}=\min _{\left(p_{t}, q_{t}\right)}\left\{A^{L}\left(t, p_{t}, q_{t}\right): p_{t}, q_{t} \text { satisfy }(3.17)\right\}
$$

The proof of Lemma 3.4 is presented in Section 7.2. To find the minimizers $\left(p_{t}, q_{t}\right)$ in $(3.16)$ we use the method of Lagrange multipliers taking into account the free boundary conditions naturally appearing. To this end, we define

$$
L^{\lambda}\left(t, p_{t}, q_{t}, p_{t}^{\prime}, q_{t}^{\prime}\right) \triangleq L\left(t, p_{t}, q_{t}, p_{t}^{\prime}, q_{t}^{\prime}\right)+\lambda(t)\left[q_{t}^{\prime}+g^{\prime}\left(\operatorname{csch}\left(\frac{\epsilon t}{\sigma}\right) p_{t}^{\prime}\right)\right]
$$

where $\lambda(t)$ designates the time dependent Lagrange multiplier. We first obtain the following lemma.

Lemma 3.5. Consider the functional

$$
\mathcal{J}\left(p_{t}, q_{t}\right) \triangleq \int_{0}^{T} L^{\lambda}\left(t, p_{t}, q_{t}, p_{t}^{\prime}, q_{t}^{\prime}\right) d t
$$

where $L^{\lambda}$ has been defined in Eq. (3.21). The minimizer $\left(p_{t}, q_{t}\right)$ of $\mathcal{J}$ satisfies

$$
\begin{gathered}
\frac{\partial L}{\partial p_{t}}-\frac{d}{d t}\left(\frac{\partial L}{\partial p_{t}^{\prime}}\right)-\frac{d}{d t}\left(\lambda(t) g^{\prime \prime}\left(\operatorname{csch}\left(\frac{\epsilon t}{\sigma}\right) p_{t}^{\prime}\right) \operatorname{csch}\left(\frac{\epsilon t}{\sigma}\right)\right)=0 \\
\frac{\partial L}{\partial q_{t}}-\frac{d}{d t}\left(\frac{\partial L}{\partial q_{t}^{\prime}}\right)-\lambda^{\prime}(t)=0 \\
\left.\frac{\partial L}{\partial p_{t}^{\prime}}\right|_{t=T}+\lambda(T) g^{\prime \prime}\left(\operatorname{csch}\left(\frac{\epsilon T}{\sigma}\right) p_{T}^{\prime}\right) \operatorname{csch}\left(\frac{\epsilon T}{\sigma}\right)=0 \\
\left.\frac{\partial L}{\partial q_{t}^{\prime}}\right|_{t=0}+\lambda(0)=0 \\
q_{t}^{\prime}=-g^{\prime}\left(\operatorname{csch}\left(\frac{\epsilon t}{\sigma}\right) p_{t}^{\prime}\right), \quad t>0
\end{gathered}
$$

The proof of Lemma 3.5 is presented in Section 7.2.

In what follows, we assume that

$$
g(t)=\xi \frac{t^{2}}{2}
$$


Remark: The assumption of quadratic penalty function is only done to get explicit expressions for the optimal action and contract. A similar analysis can be carried out with a different choice of penalty function as long as it satisfies assumption (3.1).

Eq. (3.28) turns (3.27) into

$$
p_{t}^{\prime}=-\frac{1}{\xi} \sinh \left(\frac{\epsilon t}{\sigma}\right) q_{t}^{\prime} \quad(t>0) .
$$

Note that by using Eqs. (3.4), (3.5) and (3.17), we may rewrite Eq. (3.6) as

$$
\alpha_{t}=-\frac{\sigma^{2}}{v_{t}} q_{t}^{\prime}+q_{t}
$$

In what follows, we set

$$
\beta \triangleq\left(\frac{\epsilon^{2} \xi\left(\gamma_{1}+\gamma_{2}\right)}{\left(\gamma_{1}+\gamma_{2}\right) \sigma^{2} \xi+1}\right)^{1 / 2}
$$

Employing Lemma 3.5 we obtain the following key result.

Proposition 3.2. The (unique) minimizer of $\left(p_{t}^{*}, q_{t}^{*}\right)$ of the functional $A^{L}$ defined by Eq. (3.18) is given by

$$
q_{t}^{*}=\frac{\gamma_{1}}{\gamma_{1}+\gamma_{2}} \operatorname{sech}(\beta T) \cosh (\beta t)+\frac{\gamma_{2}}{\gamma_{1}+\gamma_{2}}-\operatorname{sech}\left(\frac{\epsilon T}{\sigma}\right) \cosh \left(\frac{\epsilon t}{\sigma}\right)
$$

and

$$
\begin{aligned}
p_{t}^{*}= & \frac{\beta \gamma_{1} \sigma \operatorname{sech}(\beta T)\left(-\epsilon \cosh \left(\frac{\epsilon t}{\sigma}\right) \sinh (\beta t)+\beta \sigma \cosh (\beta t) \sinh \left(\frac{\epsilon t}{\sigma}\right)\right)}{\left(\gamma_{1}+\gamma_{2}\right)\left(\epsilon^{2}-\beta^{2} \sigma^{2}\right) \xi} \\
& +\frac{\operatorname{sech}\left(\frac{\epsilon T}{\sigma}\right)\left(\sigma \sinh \left(\frac{2 \epsilon t}{\sigma}\right)-2 \epsilon t\right)}{4 \xi \sigma}
\end{aligned}
$$

The proof of Proposition 3.2 is presented in Section 7.2. We are now ready to state the theorem giving the optimal contract and misreporting action.

Theorem 3.1. The (unique) optimal misreporting action $a_{t}^{H A}$ is represented by

$$
a_{t}^{H A}=-\frac{\gamma_{1} \beta}{\left(\gamma_{1}+\gamma_{2}\right) \xi} \operatorname{sech}(\beta T) \sinh (\beta t)+\frac{\epsilon}{\sigma \xi} \operatorname{sech}\left(\frac{\epsilon T}{\sigma}\right) \sinh \left(\frac{\epsilon t}{\sigma}\right) .
$$

Moreover, the corresponding $\alpha_{t}^{H A}$ is uniquely given by

$$
\alpha_{t}^{H A}=\frac{\gamma_{2}}{\gamma_{1}+\gamma_{2}}+\frac{\gamma_{1}}{\gamma_{1}+\gamma_{2}} \frac{\operatorname{sech}(\beta T)}{\epsilon \tanh \left(\frac{\epsilon t}{\sigma}\right)}\left[-\beta \sigma \sinh (\beta t)+\epsilon \cosh (\beta t) \tanh \left(\frac{\epsilon t}{\sigma}\right)\right]
$$

and the optimal contract can be expressed as

$$
C_{T}=-\frac{1}{\gamma_{1}} \log \left(-R_{0}\right)+d+\int_{0}^{T} \alpha_{t}^{H A} d \hat{x}_{t}^{0}
$$

where $d \triangleq d_{1}+d_{2}+d_{3}$ is the constant that makes the manager's expected utility equal to his reservation 
value, with

$$
\begin{aligned}
d_{1} \triangleq & \frac{\gamma_{1}}{2} \int_{0}^{T} \alpha_{t}^{2} \frac{v_{t}^{2}}{\sigma^{2}} d t \\
= & \frac{\gamma_{1}\left(\beta T \gamma_{1}^{2}\left(\epsilon^{2}-\beta^{2} \sigma^{2}\right) \operatorname{sech}^{2}(\beta T)+\gamma_{1}\left(\epsilon^{2}\left(\gamma_{1}+4 \gamma_{2} \epsilon\right)+\beta^{2} \gamma_{1} \sigma^{2}\right) \tanh (\beta T)\right)}{4 \beta\left(\gamma_{1}+\gamma_{2}\right)^{2}} \\
& +\frac{\gamma_{1}\left(2 \beta \epsilon\left(\gamma_{2}^{2} T \epsilon-\left(\gamma_{1}+\gamma_{2}\right)^{2} \sigma \tanh \left(\frac{\epsilon T}{\sigma}\right)\right)\right)}{4 \beta\left(\gamma_{1}+\gamma_{2}\right)^{2}}
\end{aligned}
$$

and

$$
d_{2} \triangleq \int_{0}^{T} \alpha_{t} \frac{v_{t}}{\sigma}\left(-\frac{\sigma \theta \epsilon}{v_{t}}-\frac{a_{t}^{H A}}{\sigma}+\frac{\hat{x}_{t}^{0}-\hat{x}_{t}^{a^{H A}}}{\sigma}\right) d t
$$

and

$$
\begin{aligned}
d_{3} \triangleq & \int_{0}^{T} \frac{\xi}{2}\left(a_{t}^{H A}\right)^{2} d t \\
= & \frac{\beta \gamma_{1}^{2} \operatorname{sech}^{2}(\beta T)(\sinh (2 \beta T)-2 \beta T)}{8 \xi\left(\gamma_{1}+\gamma_{2}\right)^{2}}+\frac{\epsilon\left(\sigma \tanh \left(\frac{\epsilon T}{\sigma}\right)-\epsilon T \operatorname{sech}^{2}\left(\frac{\epsilon T}{\sigma}\right)\right)}{4 \xi \sigma^{2}} \\
& +\frac{\beta \gamma_{1} \epsilon\left(\beta \sigma \tanh \left(\frac{\epsilon T}{\sigma}\right)-\epsilon \tanh (\beta T)\right)}{8 \xi\left(\gamma_{1}+\gamma_{2}\right)\left(\epsilon^{2}-\beta^{2} \sigma^{2}\right)}
\end{aligned}
$$

where $p_{t}^{*}$ is defined in (3.32).

Proof. For $0 \leq t \leq T$, we set $p_{t}^{*}$ and $q_{t}^{*}$ as in (3.4) and (3.5). By the application of Lemma 3.4 and Proposition 3.2, we have that $a_{t}^{H A}$ minimizes the functional $A\left(t, a_{t}\right)$ as long as the pair $\left(p_{t}^{*}, q_{t}^{*}\right)$ solves the optimization (minimization) problem defined by Eq. (3.22) and Eq. (3.23)-(3.27). Application of Lemma 3.2 yields that the solution $q_{t}^{*}$ is given by Eq. (3.31). Differentiating the expression for $q_{t}^{*}$ given in Eq. (3.31), we obtain Eq. (7.52), which immediately implies that the optimal misreporting action $a_{t}^{H A}$ is

$$
a_{t}^{H A}=-\frac{1}{\xi}\left(q_{t}^{*}\right)^{\prime}=-\frac{\gamma_{1} \beta}{\left(\gamma_{1}+\gamma_{2}\right) \xi} \operatorname{sech}(\beta T) \sinh (\beta t)+\frac{\epsilon}{\sigma \xi} \operatorname{sech}\left(\frac{\epsilon T}{\sigma}\right) \sinh \left(\frac{\epsilon t}{\sigma}\right)
$$

Plugging the expression for $a_{t}^{H A}$ inside Eq. (3.6), we obtain after straightforward calculations that the optimal $\alpha_{t}^{H A}$ is

$$
\begin{aligned}
\alpha_{t}^{H A} & =-\frac{\sigma^{2}}{v_{t}}\left(q_{t}^{*}\right)^{\prime}+q_{t}^{*} \\
& =\frac{\gamma_{1}}{\gamma_{1}+\gamma_{2}} \frac{\operatorname{sech}(\beta T)}{\epsilon \tanh \left(\frac{\epsilon t}{\sigma}\right)}\left[-\beta \sigma \sinh (\beta t)+\epsilon \cosh (\beta t) \tanh \left(\frac{\epsilon t}{\sigma}\right)\right]+\frac{\gamma_{2}}{\gamma_{1}+\gamma_{2}}
\end{aligned}
$$


Using the expression for $\alpha_{t}^{H A}$ we obtain

$$
\int_{0}^{T} \alpha_{t}^{H A} d \hat{x}_{t}^{0}=\int_{0}^{T}\left(\frac{\gamma_{1}}{\gamma_{1}+\gamma_{2}} \frac{\operatorname{sech}(\beta T)}{\epsilon \tanh \left(\frac{\epsilon t}{\sigma}\right)}\left[-\beta \sigma \sinh (\beta t)+\epsilon \cosh (\beta t) \tanh \left(\frac{\epsilon t}{\sigma}\right)\right]+\frac{\gamma_{2}}{\gamma_{1}+\gamma_{2}}\right) d \hat{x}_{t}^{0}
$$

Recall that the expression for the optimal contract is given by Eq. (3.12). Using the expression for $\alpha_{t}^{H A}$, we obtain that

$$
\frac{\gamma_{1}}{2} \int_{0}^{T} \alpha_{t}^{2} \frac{v_{t}^{2}}{\sigma^{2}} d t
$$

evaluates to $d_{1}$ given in Eq. (3.36). Expressing Eq. (2.12) in terms of d $\hat{Z}_{t}^{a}$, for any function $a_{t}$, we obtain

$$
d \hat{Z}_{t}^{a}=\frac{\sigma}{v_{t}} d \hat{x}_{t}^{0}-\frac{\sigma \theta \epsilon}{v_{t}} d t-\frac{a_{t}}{\sigma} d t+\frac{\hat{x}_{t}^{0}-\hat{x}_{t}^{a}}{\sigma} d t
$$

The expression for $\hat{x}_{t}^{0}-\hat{x}_{t}^{a}$ has been derived in Eq. (2.12) and so we use

$$
p_{t}^{*}=\int_{0}^{t} \sinh \left(\frac{\epsilon s}{\sigma}\right) a_{s}^{H A} d s
$$

given in Eq. (3.32) to compute $\frac{\hat{x}_{t}^{0}-\hat{x}_{t}^{a^{H A}}}{\sigma}$. Consequently, we have

$$
\int_{0}^{T} \alpha_{t} \frac{v_{t}}{\sigma}\left(-\frac{\sigma \theta \epsilon}{v_{t}}-\frac{a_{t}^{H A}}{\sigma}-\frac{\hat{x}_{t}^{0}-\hat{x}_{t}^{a^{H A}}}{\sigma}\right) d t
$$

leading to $d_{2}$ in Eq. (3.37). Next, we compute

$$
G_{T}=\int_{0}^{T} \frac{\xi}{2}\left(a_{t}^{H A}\right)^{2} d t
$$

yielding $d_{3}$ given in Eq. (3.38). Altogether, we obtain that the optimal contract is given by Eq. (3.35).

Remark 3.2. Verification of Assumptions 2.1 and 3.2. It can be easily verified that Assumption 2.1 is satisfied, given that both $a_{t}^{H A}$ and $\alpha_{t}^{H A}$ are smooth (infinitely differentiable) functions. This results from the fact that $q_{t}^{*}$ and $p_{t}^{*}$ obtained in (3.31) and (3.32) are smooth, and thus all calculations carried out in this section are fully justified. We next show that Assumption 3.2 is satisfied. Observe that

$$
\beta^{2}=\frac{\left(\gamma_{1}+\gamma_{2}\right) \epsilon^{2}}{\frac{1}{\xi}+\left(\gamma_{1}+\gamma_{2}\right) \sigma^{2}}<\frac{\left(\gamma_{1}+\gamma_{2}\right) \epsilon^{2}}{\left(\gamma_{1}+\gamma_{2}\right) \sigma^{2}}=\frac{\epsilon^{2}}{\sigma^{2}}
$$

Notice that since $\beta \sigma \leq \epsilon$, we observe that $\tanh (\beta t) \leq \tanh \left(\frac{\epsilon t}{\sigma}\right)$ and so, for all $t \geq 0$, we have

$$
\sinh (\beta t)=\cosh (\beta t) \tanh (\beta t) \leq \cosh (\beta t) \tanh \left(\frac{\epsilon t}{\sigma}\right) .
$$


Therefore,

$$
\begin{aligned}
\alpha_{t}^{H A} & \geq \frac{\gamma_{2}}{\gamma_{1}+\gamma_{2}} \frac{\operatorname{sech}(\beta T)}{\tanh \left(\frac{\epsilon t}{\sigma}\right)}(\sinh (\beta t)-\sinh (\beta t))+\frac{\gamma_{2}}{\gamma_{1}+\gamma_{2}} \\
& =\frac{\gamma_{2}}{\gamma_{1}+\gamma_{2}}
\end{aligned}
$$

from which we infer that $\alpha_{t}^{H A} \geq 0$ for all $t \geq 0$, and thus Assumption 3.2 is satisfied.

\section{Risk-Sharing}

We now study the risk-sharing problem of maximizing the joint welfare

$$
J(T):=J_{1}(T)+\lambda J_{2}(T)=\mathbb{E}^{\mathbb{P}}\left[U_{1}\left(C_{T}-G_{T}\right)\right]+\lambda \mathbb{E}^{\mathbb{P}}\left[U_{2}\left(\hat{x}_{T}^{0}-C_{T}\right)\right],
$$

where $\lambda$ is a given constant representing the relative risk-sharing level. We maintain the assumption that both $U_{1}(x)$ and $U_{2}(x)$ are exponential and given by Eq. (3.3). The above is a classical risksharing problem, or joint welfare problem, resulting in the so-called first-best solution. Here, in addition to sharing $C_{T}$, the shareholders and the manager also have to find the jointly optimal choice of the misreporting level $a$, which can be contracted upon. Differently from the hidden action case, we don't restrict the contracts to be linear, rather, we find the optimal contract to be linear. More precisely, taking a derivative with respect to $C_{T}$ inside the expectation and setting it equal to zero gives us the classical Borch rule for risk-sharing, that the ratio of the marginal utilities is constant:

$$
U_{1}^{\prime}\left(C_{T}-G_{T}\right)=\lambda U_{2}^{\prime}\left(\hat{x}_{T}^{0}-C_{T}\right) .
$$

As the utilities functions of shareholders and manager are exponential, we get the following expression for the optimal risk-sharing contract, for an appropriate constant $c$,

$$
C_{T}=c+\alpha^{R S} \hat{x}_{T}^{0}+\frac{\gamma_{1}}{\gamma_{1}+\gamma_{2}} G_{T}
$$

where

$$
\alpha^{R S}=\frac{\gamma_{2}}{\gamma_{1}+\gamma_{2}}
$$

As we only allow deterministic control actions $a_{t}, G_{T}$ is deterministic. Thus, the above expression for $C_{T}$ is in agreement with a standard result that shows that the optimal risk-sharing fraction is determined by the relative size of the risk aversion parameters $\gamma_{1}, \gamma_{2}$. We also see that the fact that the outcome process is not observed does not play a role here, that is, the PPS does not depend on the observation noise $\sigma$. Compared to the previous hidden action case, PPS here agrees with PPS there in the special case of $\sigma$ or $\xi$ being infinite (see also Section 5 for more details). Under the probability measure $\mathbb{P}$, the dynamics of the model are

$$
\begin{aligned}
d x_{t} & =\theta \epsilon d t+\epsilon d W_{t} \\
d y_{t} & =\left[x_{t}+a_{t}\right] d t+\sigma d Z_{t}
\end{aligned}
$$


where $Z_{t}$ is a $\mathcal{F}_{t}$-Brownian motion. The information structure is the same as in the hidden action case. The dynamics of $\hat{x}_{t}$ are given by the Kalman-Bucy filter as

$$
d \hat{x}_{t}=\theta \epsilon d t+\frac{v_{t}}{\sigma} d \hat{Z}_{t}
$$

where $\hat{Z}_{t}$ is the filter innovation process, a $\left(\mathcal{F}_{t}^{y}, \mathbb{P}\right)$-Brownian motion. The market, instead, does not know the exact level of misreporting, and believes that the observation model is

$$
\begin{aligned}
& d x_{t}=\theta \epsilon d t+\epsilon d W_{t} \\
& d y_{t}=x_{t} d t+\sigma d Z_{t}^{0}
\end{aligned}
$$

where $d Z_{t}^{0}=d Z_{t}+\frac{a_{t}}{\sigma} d t$. By an analogous reasoning used in the derivation of Eq. (2.11), we obtain

$$
\hat{Z}_{t}=\hat{Z}_{t}^{0}-\int_{0}^{t} \frac{a_{s}}{\sigma} d s+\int_{0}^{t} \frac{\hat{x}_{s}^{0}-\hat{x}_{s}}{\sigma} d s
$$

and, consequently, $\hat{x}_{t}^{0}$ may be expressed in terms of $\hat{Z}_{t}$ as

$$
d \hat{x}_{t}^{0}=\epsilon \theta d t+\frac{v_{t}}{\sigma} d \hat{Z}_{t}+\frac{v_{t} a_{t}}{\sigma^{2}} d t-\frac{v_{t}}{\sigma^{2}}\left(\hat{x}_{t}^{0}-\hat{x}_{t}\right) d t
$$

Using (4.2) and substituting back into $J(T)$, we see that shareholders and manager maximize over $a$ the expression

$$
-\left(1+\frac{\gamma_{1}}{\gamma_{2}}\right) \mathbb{E}\left[\exp \left\{-\gamma_{1} c-\frac{\gamma_{1} \gamma_{2}}{\gamma_{1}+\gamma_{2}}\left(\hat{x}_{T}^{0}-G_{T}\right)\right\}\right] .
$$

This is now a standard stochastic control problem, for which it is known that the optimal control $a_{t}$ is deterministic (and it can be verified by solving the HJB equation). In order to solve it, denote

$$
\Gamma=\frac{\gamma_{1} \gamma_{2}}{\gamma_{1}+\gamma_{2}}
$$

For concreteness, we assume again quadratic penalty $a$, i.e.

$$
G_{T}=\xi \int_{0}^{T} \frac{a_{t}^{2}}{2} d t
$$

Then, the optimization problem boils down to maximizing over $a_{t} \geq 0$

$$
I\left(a_{t}\right)=\int_{0}^{T}\left\{\theta \epsilon+\frac{1}{\sigma^{2}} a_{t} v_{t}-\xi \frac{a_{t}^{2}}{2}-\frac{1}{2} \Gamma \frac{v_{t}^{2}}{\sigma^{2}}-\frac{v_{t}}{\sigma} \frac{\hat{x}_{t}^{0}-\hat{x}_{t}}{\sigma}\right\} d t
$$

which, by Eq. (2.12), simplifies to

$$
I\left(a_{t}\right)=\int_{0}^{T}\left\{\theta \epsilon+\frac{1}{\sigma^{2}} a_{t} v_{t}-\xi \frac{a_{t}^{2}}{2}-\frac{1}{2} \Gamma \frac{v_{t}^{2}}{\sigma^{2}}-\frac{\epsilon}{\sigma^{2}} \frac{v_{t} \int_{0}^{t} \sinh \left(\frac{\epsilon s}{\sigma}\right) a_{s} d s}{\sigma \cosh \left(\frac{\epsilon t}{\sigma}\right)}\right\} d t .
$$

We make the following

Assumption 4.3. $a_{t}$ is non-negative and continuously differentiable in the interval $[0, T]$. 
Set

$$
p_{t} \triangleq \int_{0}^{t} \sinh \left(\frac{\epsilon s}{\sigma}\right) a_{s} d s \geq 0
$$

as before. Then

$$
p_{t}^{\prime}=\sinh \left(\frac{\epsilon t}{\sigma}\right) a_{t} .
$$

Moreover, $p_{0}=0$ and $p_{T}$ is a free boundary condition. Maximizing $I\left(a_{t}\right)$ over $a_{t}$ amounts to minimizing

$$
\tilde{I}\left(p_{t}\right) \triangleq \int_{0}^{T} \tilde{L}\left(t, p_{t}, p_{t}^{\prime}\right) d t
$$

where

$$
\begin{aligned}
\tilde{L}\left(t, p_{t}, p_{t}^{\prime}\right) \triangleq & -\theta \epsilon-\frac{\epsilon}{\sigma} \operatorname{sech}\left(\frac{\epsilon t}{\sigma}\right) p_{t}^{\prime}+\frac{\xi}{2}\left(\operatorname{csch}\left(\frac{\epsilon t}{\sigma}\right)\right)^{2}\left(p_{t}^{\prime}\right)^{2} \\
& +\frac{1}{2} \epsilon^{2} \Gamma\left(\tanh \left(\frac{\epsilon t}{\sigma}\right)\right)^{2}+\frac{\epsilon^{2}}{\sigma^{2}} \tanh \left(\frac{\epsilon t}{\sigma}\right) \operatorname{sech}\left(\frac{\epsilon t}{\sigma}\right) p_{t}
\end{aligned}
$$

Lemma 4.6. There is a minimizer $p_{t}$ of $\tilde{I}$ defined in (4.12) and maximizer $a_{t}$ of $I\left(a_{t}\right)$ defined in (4.10).

The proof of Lemma 4.6 is presented in Section 7.3. We also have the following

Lemma 4.7. The minimizer $p_{t}$ of $\tilde{I}$ is given by

$$
p_{t}=\frac{\operatorname{sech}\left(\frac{\epsilon T}{\sigma}\right)}{4 \sigma \xi}\left(\sigma \sinh \left(\frac{2 \epsilon t}{\sigma}\right)-2 \epsilon t\right) .
$$

The proof of Lemma 4.7 is presented in Section 7.3. Using Lemma 4.6 and Lemma 4.7 we end up with

Theorem 4.2. The maximizer $a_{t}^{R S}$ of $I\left(a_{t}\right)$ in (4.10) is

$$
a_{t}^{R S}=\frac{\epsilon}{\xi \sigma} \operatorname{sech}\left(\frac{\epsilon T}{\sigma}\right) \sinh \left(\frac{\epsilon t}{\sigma}\right) .
$$

Moreover, the optimal contract is given by

$$
C_{T}=c+\alpha^{R S} \hat{x}_{T}^{0}+\frac{\gamma_{1}}{\gamma_{1}+\gamma_{2}} \frac{\epsilon}{4 \xi \sigma^{2}}\left(\sigma \tanh \left(\frac{\epsilon T}{\sigma}\right)-\epsilon T \operatorname{sech}\left(\frac{\epsilon T}{\sigma}\right)^{2}\right) .
$$

Proof. Differentiating $p_{t}$ in Eq. (4.14), we obtain (7.55), thus we immediately arrive at Eq. (4.15). Plugging the derived expression for $a_{t}^{R S}$ inside Eq. (4.2), we obtain the optimal contract as in Eq. (4.16).

Remark 4.3. Verification of Assumption 4.3. Looking at Eq. (4.15), we can immediately see that $a_{t}^{R S}$ is an infinitely differentiable function, and therefore continuously differentiable.

Let us now denote by $V$ the following quantity

$$
V=\exp \left\{\frac{K_{1}+K_{2}+K_{3}}{4\left(\gamma_{1}+\gamma_{2}\right)^{2} \sigma^{2} \xi}\right\}
$$


where

$$
\begin{gathered}
K_{1}=\gamma_{1} \gamma_{2}\left(-T\left(\gamma_{1}+\gamma_{2}-2\right)\left(\gamma_{1}+\gamma_{2}\right) \epsilon^{2} \operatorname{sech}^{2}\left(\frac{T \epsilon}{\sigma}\right)\right) \\
K_{2}=\gamma_{1} \gamma_{2} \sigma\left(-2\left(2 x_{0}\left(\gamma_{1}+\gamma_{2}\right)+\epsilon T\left(2 \theta\left(\gamma_{1}+\gamma_{2}\right)-\gamma_{1} \gamma_{2} \epsilon\right)\right)\right) \sigma \xi \\
K_{3}=\gamma_{1} \gamma_{2} \sigma \epsilon\left(\gamma_{1}^{2}+\left(\gamma_{2}-2\right) \gamma_{2}-2 \gamma_{1}\left(1-\gamma_{2}+\gamma_{2} \sigma^{2} \xi\right) \tanh \left(\frac{\epsilon T}{\sigma}\right)\right)
\end{gathered}
$$

Plugging Eq. (4.16) and Eq. (4.15) into the manager utility, we find that

$$
J_{1}(T)=-e^{-\gamma_{1} c} V
$$

Similarly, we obtain that, at the optimum, the shareholders utility is given by

$$
J_{2}(T)=-e^{\gamma_{2} c} V
$$

Using Eq. (4.1), we can then deduce that the constant $c$ is given by

$$
c=\frac{1}{\gamma_{1}+\gamma_{2}} \log \left(\frac{\gamma_{1}}{\lambda \gamma_{2}}\right) \text {. }
$$

\section{Comparative Statics}

In this section we discuss, from the economical perspective, the results obtained in the earlier sections. Section 5.1 describes the optimal PPS and misreporting level as functions of time, Section 5.2 analyzes the optimal contract, Section 5.3 does a comparative statics analysis for the optimal misreporting action, and Section 5.4 compares the shareholders and manager utilities in hidden action and risksharing settings.

\subsection{Pay-per-performance sensitivity and misreporting}

We study the dependence on time of the optimal pay-per-performance sensitivity and the optimal misreporting. Figure 1 shows that they both increase over time. Therefore, the shareholders find it optimal to induce the manager to gradually increase the level of misreporting over time, and they achieve that by offering him a higher pay-per-performance sensitivity as time progresses. This suggests that in a context in which outside investors cannot correctly infer the level of misreporting, early auditing will prevent the occurrence of high levels of accounting irregularities.

\subsection{Contracts}

Differently from Holmstrom and Milgrom (1987), in the hidden action case we find that noisy observations lead to contracts which are dependent on the full path of the observation process. In the risk-sharing case, instead, the pay-per-performance sensitivity only depends on the relative size of the risk aversion parameters $\gamma_{1}$ and $\gamma_{2}$, and, additionally, it is independent of $\sigma$. Thus, the fact that the actual outcome process is unobserved does not play any role. Comparing the expressions for the optimal pay-per-performance sensitivity obtained in the hidden action case (see Eq. (3.34)) and risk-sharing case (see Eq. (4.3)), we obtain

$$
\alpha_{t}^{H A}=\alpha_{t}^{R S}+\frac{\gamma_{1}}{\gamma_{1}+\gamma_{2}} \frac{\operatorname{sech}(\beta T)}{\epsilon \tanh \left(\frac{\epsilon t}{\sigma}\right)}\left[-\beta \sigma \sinh (\beta t)+\epsilon \cosh (\beta t) \tanh \left(\frac{\epsilon t}{\sigma}\right)\right]
$$



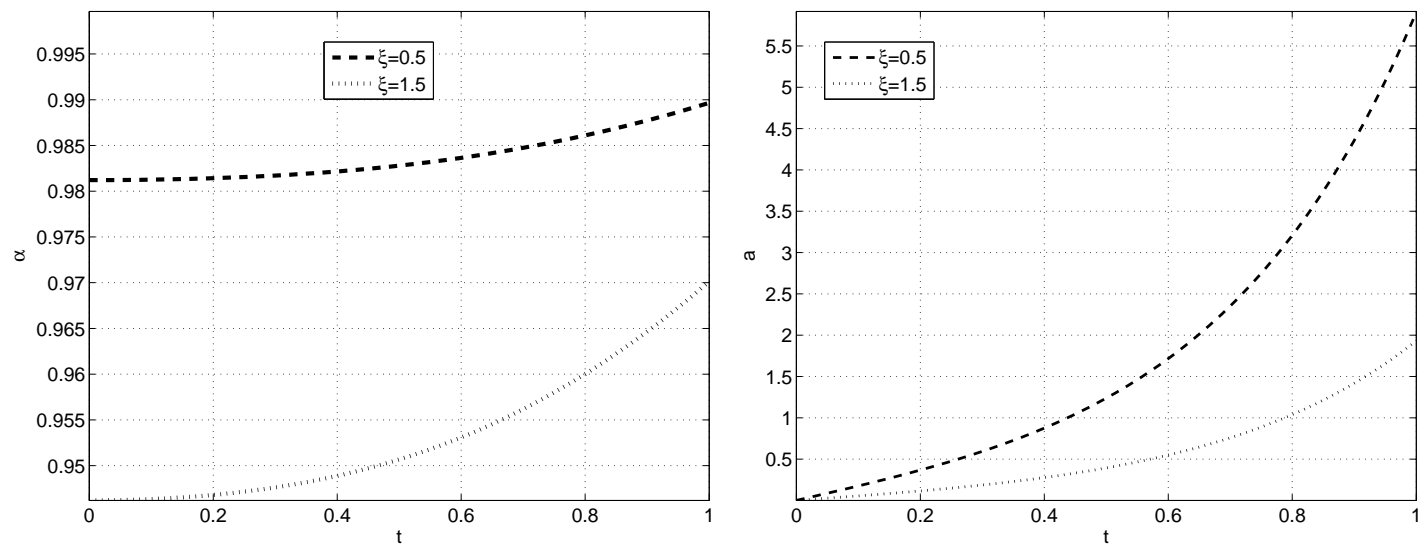

Figure 1: The parameters common to all graphs are $\sigma=0.1, \epsilon=0.3, \gamma_{1}=0.7, \gamma_{2}=0.3, T=1$. The left graph refers to the pay-per-performance sensitivity over time, while the right graph to the misreporting level over time.

where we recall that $\alpha_{t}^{H A}$ and $\alpha_{t}^{R S}$ denote, respectively, the pay-per-performance sensitivity in the hidden action and risk-sharing setting. As shown in Eq. (3.46), we have $\alpha_{t}^{H A}>\alpha_{t}^{R S}$. This is a familiar result from existing models: when action cannot be contracted upon, the manager needs extra incentives from the shareholders in terms of compensation in order to apply desired action. We also find

$$
\begin{aligned}
\lim _{\epsilon \rightarrow 0} \alpha_{t}^{H A} & =1 \\
\lim _{\gamma_{1} \rightarrow 0} \alpha_{t}^{H A} & =\lim _{\gamma_{1} \rightarrow 0} \alpha_{t}^{R S}=1
\end{aligned}
$$

Thus, if the manager does not care about risk the whole firm is transferred to him, a familiar result in the existing literature. In the hidden action case, this also happens in the case when the outcome process becomes constant and known to everyone, $\epsilon \approx 0$. (In this case there is no risk to anyone, and zero action, so only non-risky cash amount is divided, and it does not really matter who owns the firm). We also have

$$
\begin{aligned}
\lim _{\sigma \rightarrow 0} \alpha_{t}^{H A} & =\frac{\gamma_{2}}{\gamma_{1}+\gamma_{2}}+\frac{\gamma_{1}}{\gamma_{1}+\gamma_{2}} \cosh \left(\epsilon^{2} \xi t\left(\gamma_{1}+\gamma_{2}\right)\right) \operatorname{sech}\left(\epsilon^{2} \xi T\left(\gamma_{1}+\gamma_{2}\right)\right) \\
\lim _{\sigma \rightarrow \infty} \alpha_{t}^{H A} & =\frac{\gamma_{2}}{\gamma_{1}+\gamma_{2}} .
\end{aligned}
$$

Therefore, for low levels of accounting noise the pay-per-performance sensitivity in the hidden action setting remains strictly higher than in the risk-sharing setting (this is why, as we shall see below, the optimal hidden action becomes negative for small $\sigma$ ). For high levels of accounting noise, instead, we have that the pay-per-performance is the same in risk-sharing and hidden action case. This is not surprising, as the infinite noise impedes any possibility of giving meaningful incentives. We also 

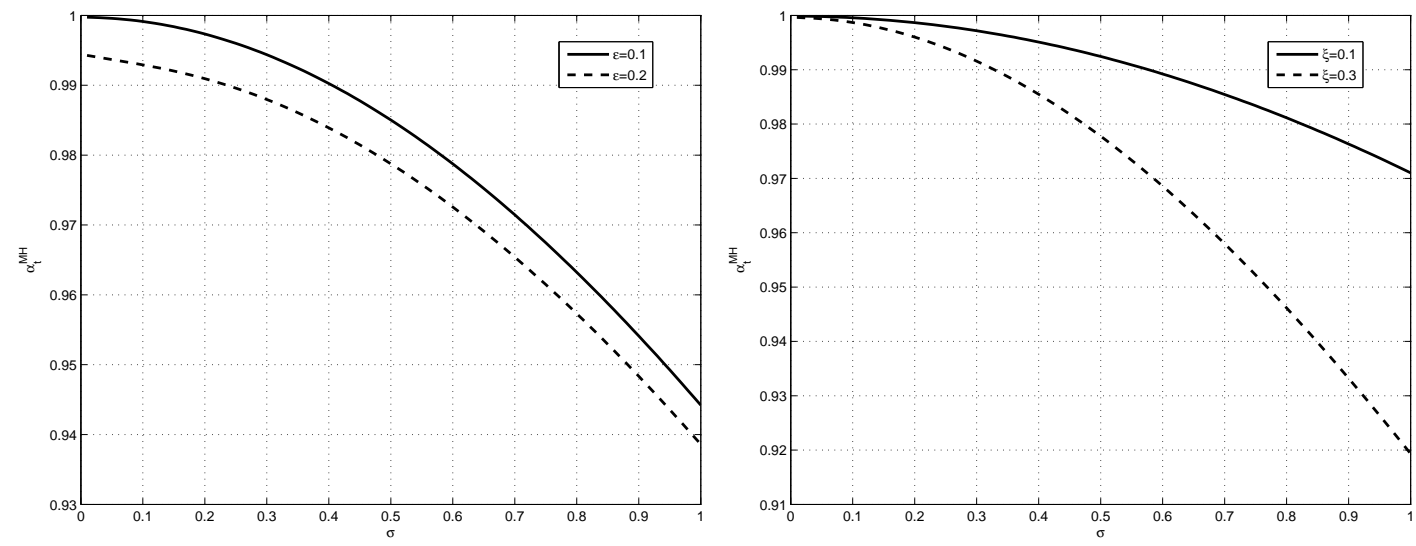

Figure 2: Left panel: $\alpha_{t}^{H A}$ versus $\sigma$ for two different levels of $\epsilon, \epsilon=0.1$, and $\epsilon=0.2$. The parameter choices are $\gamma_{1}=0.3, \gamma_{2}=0.1, \xi=0.2, t=0.5, T=1$. Right panel: $\alpha_{t}^{H A}$ versus $\sigma$ for two different misreporting penalties, $\xi=0.1$, and $\xi=0.3$. The parameter choices are $\gamma_{1}=0.3, \gamma_{2}=0.1, \epsilon=0.1$, $t=0.5, T=1$.

find

$$
\begin{aligned}
\lim _{\xi \rightarrow 0} \alpha_{t}^{H A} & =1 \\
\lim _{\xi \rightarrow \infty} \alpha_{t}^{H A} & =\alpha_{t}^{R S} .
\end{aligned}
$$

In case of very large penalties, the pay-per-performance sensitivities are the same. This is because, in the hidden action setting, the shareholders know that the manager will not apply any misreporting action due to the high penalty incurred. On the opposite extreme, when the penalty is very small, then the shareholders know that the manager will not be blocked by severe misreporting penalties, and thus they try to give him the highest incentives to apply the misreporting action. We find that $\alpha_{t}^{H A}$ is a decreasing function of the project scale factor $\epsilon$ (proof presented in Section 7.4). This may be explained observing that, for a contract consisting of cash and a performance related compensation depending linearly on $\hat{x}_{t}^{0}$, we have that increasing $\epsilon$ is like increasing portfolio holdings of the manager in the risky asset $\hat{x}_{t}^{0}$ relative to cash. According to this interpretation, high $\alpha$ and high $\epsilon$ have the same effect on the manager, and result in the manager having higher exposure to the risky part of the compensation. Therefore, if $\epsilon$ goes up, then $\alpha_{t}^{H A}$ should go down to compensate.

It is seen from Fig. 2 that $\alpha_{t}^{H A}$ decreases as the accounting noise $\sigma$ increases. This is consistent with the fact that, as $\sigma$ increases, $\hat{x}_{t}^{0}$ becomes "closer" to $\hat{x}_{t}^{a}$, for any fixed $a$, and there is less benefit of giving to the manager incentives to apply the misreporting action. It is also seen from Fig. 3 that $\alpha_{t}^{H A}$ decreases as the penalty $\xi$ increases, since the incentives are less efficient. Moreover, PPS is much more sensitive to the risk aversion level $\gamma_{1}$ of the manager and very mildly affected by the risk aversion level $\gamma_{2}$ of the shareholders. More precisely, $\alpha_{t}^{H A}$ decreases as $\gamma_{1}$ increases, because the manager does not like risk. It increases (but at a slower rate) as the shareholders' risk aversion increases. This is because the shareholders then like risk less, and want to transfer it to the manager. 

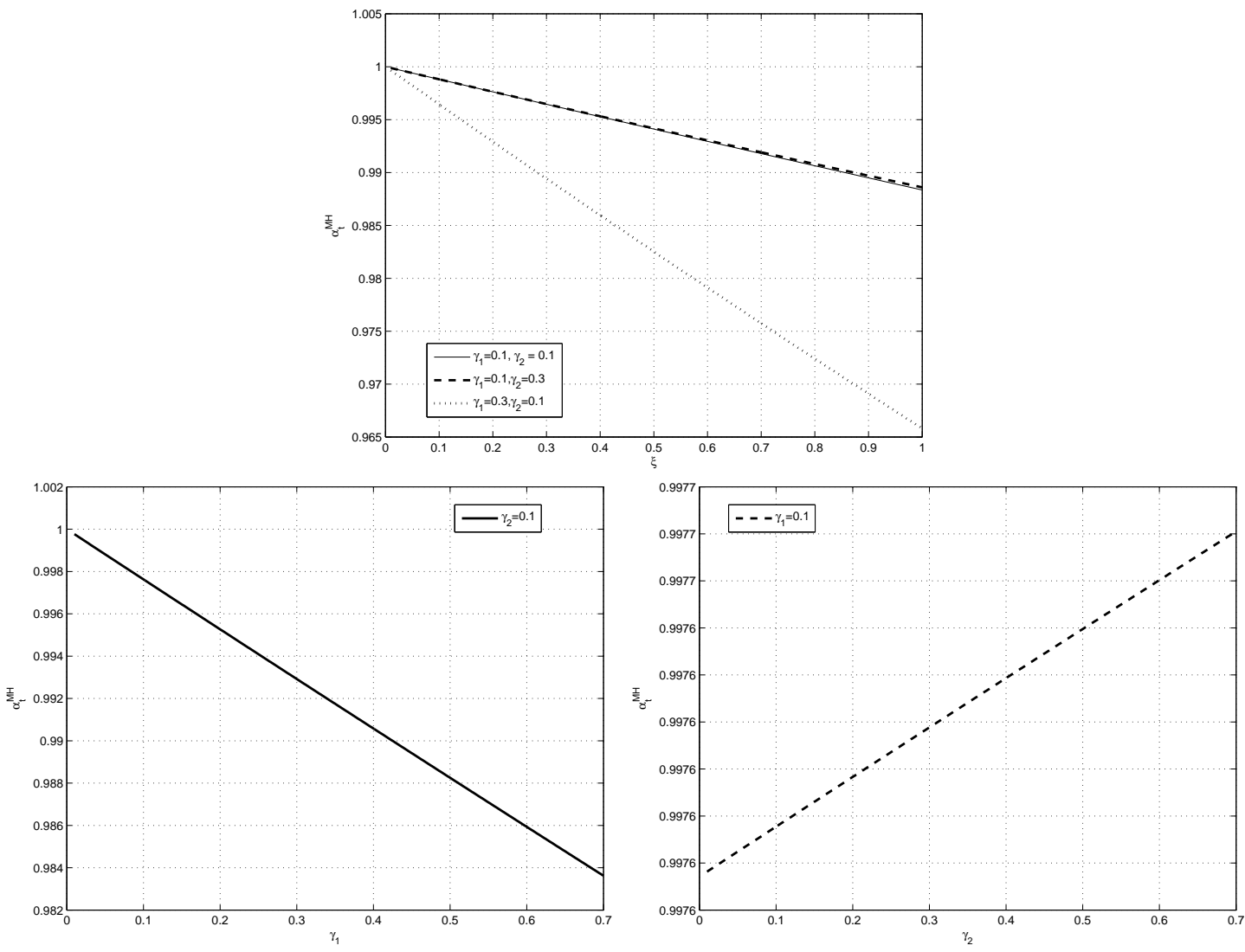

Figure 3: The parameters used in all panels are $\epsilon=0.5, \sigma=0.1, t=0.5, T=1$. Top panel: manager's misreporting level versus $\xi$ for three different choices of $\left(\gamma_{1}, \gamma_{2}\right)$. Bottom left panel: manager's misreporting level versus $\gamma_{1}$ for a fixed level of $\gamma_{2}=0.1$, and $\xi=0.1$. Bottom right panel: manager's misreporting level versus $\gamma_{2}$ for a fixed level of $\gamma_{1}=0.1$, and $\xi=0.1$. 


\subsection{Misreporting action}

We now perform analysis of the optimal misreporting action. First, as mentioned above, unlike in the classical Holmstrom and Milgrom (1987) model, the manager's optimal misreporting action is time dependent in both the risk-sharing and hidden action framework. Next, comparing the expressions for the optimal $a_{t}^{H A}$ obtained in the hidden action case (see Eq. (3.33)) and $a_{t}^{R S}$ computed in the risk-sharing case (see Eq. (4.15)), we can immediately establish that

$$
a_{t}^{H A}=a_{t}^{R S}-\frac{\gamma_{1} \beta}{\left(\gamma_{1}+\gamma_{2}\right) \xi} \operatorname{sech}(\beta T) \sinh (\beta t)
$$

Thus, we have $a_{t}^{H A}<a_{t}^{R S}$, which is expected, as in the risk-sharing case the optimal action is chosen to maximize the joint utility of shareholders and manager, whereas in the hidden action case the shareholders have to induce the manager to apply a given misreporting action by offering him an appropriate contract. Consistently with the intuition, we have that $a_{t}^{H A}$ and $a_{t}^{R S}$ are both decreasing in the size of penalty, starting from $a_{t}$ being infinity when there is zero penalty (in this case the manager would only benefit from misreporting), and converging to zero when the penalty $\xi$ goes to infinity.

We find that, for both risk-sharing and hidden action, $a_{t}^{H A}$ and $a_{t}^{R S}$ approach zero when $\sigma$ goes to infinity. This is because increase in compensation that the manager would obtain biasing the output process by $a_{t}$ would be fully absorbed by the high level of accounting noise $\sigma$. Mathematically, this can be seen from Eq. (2.12) of Lemma 2.2, showing that for a fixed misreporting action $a_{t}$,

$$
\lim _{\sigma \rightarrow \infty} \hat{x}_{t}^{0}=\hat{x}_{t}^{a} .
$$

Therefore, the manager would pay a penalty for misreporting without getting any benefit from it. In other words, "fooling the market" is not possible because the filter estimate $\hat{x}_{t}^{0}$ coincides with the market estimate.

When the observation noise is small, we obtain the following limiting behavior

$$
\begin{aligned}
\lim _{\sigma \rightarrow 0^{+}} a_{t}^{H A} & =-\frac{\gamma_{1} \epsilon}{\sqrt{\left(\gamma_{1}+\gamma_{2}\right) \xi}} \operatorname{sech}\left(\epsilon T \sqrt{\left(\gamma_{1}+\gamma_{2}\right) \xi}\right) \sinh \left(\epsilon t \sqrt{\left(\gamma_{1}+\gamma_{2}\right) \xi}\right) \\
\lim _{\sigma \rightarrow 0^{+}} a_{t}^{R S} & =0
\end{aligned}
$$

This shows that there is a tradeoff between the benefit of the manager and the shareholders. While the first always benefits from higher misreporting (given a fixed cost), the latter may lose in utility by paying the manager a higher proportion of the market value $\hat{x}_{t}^{0}$. The limits in Eq. (5.1) show that in the risk-sharing case the best joint action for small noise is not to introduce misreporting. In the hidden action case, where the shareholders induce the optimal misreporting action of the manager, we can see that it is optimal to induce him even to bias the output process downwards, with negative $a$, in which case the shareholders pay lower compensation because the market value is lower. Further analysis shows that, for a fixed $\epsilon$ (or fixed $\sigma$ ), the optimal misreporting level switches from increasing to decreasing after a given value of $\sigma$ (respectively $\epsilon$ ). This can be seen from figure 4 , which also shows that the level of $\sigma$ (respectively $\epsilon$ ), at which the misreporting amount starts decreasing depends on the project volatility $\epsilon$ (respectively $\sigma$ ), but not much on the penalty $\xi$, which mainly drives the size of the misreporting amount. We also find the following limiting behavior 

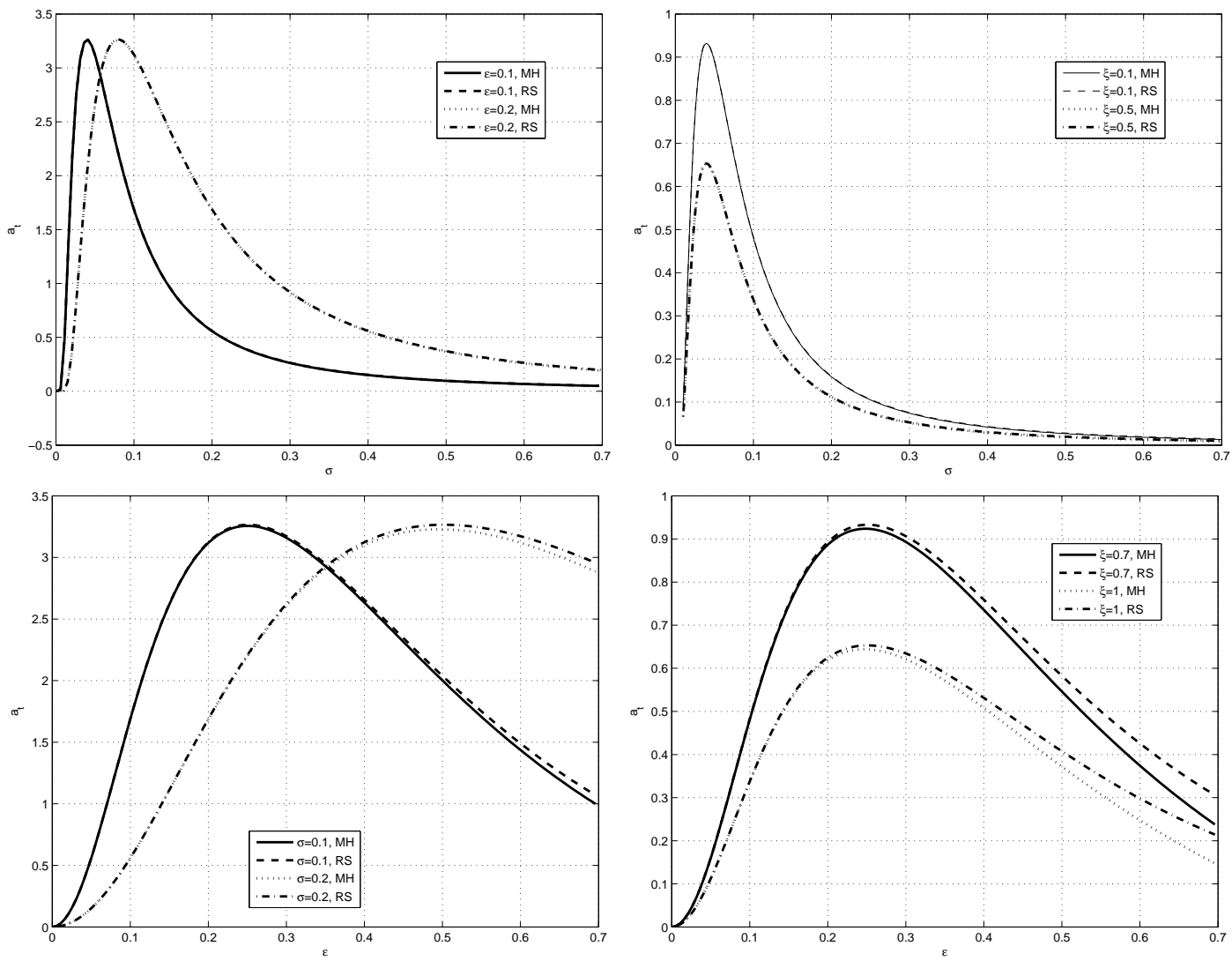

Figure 4: All plots are produced using $\gamma_{1}=0.3, \gamma_{2}=0.1, t=0.5$, and $T=1$. Top left panel: manager's misreporting level versus $\sigma$ for two different levels of $\epsilon, \epsilon=0.1$, and $\epsilon=0.2$. We fix $\xi=0.2$. Top right panel: manager's misreporting level versus $\sigma$ for two different misreporting penalties, $\xi=0.1$, and $\xi=0.5$. We fix $\epsilon=0.1$. Bottom left panel: manager's misreporting level versus $\epsilon$ for two different levels of $\sigma, \sigma=0.1$, and $\sigma=0.2$. We fix $\xi=0.2$. Top right panel: manager's misreporting level versus $\epsilon$ for two different misreporting penalties, $\xi=0.1$, and $\xi=0.5$. We fix $\sigma=0.1$. 

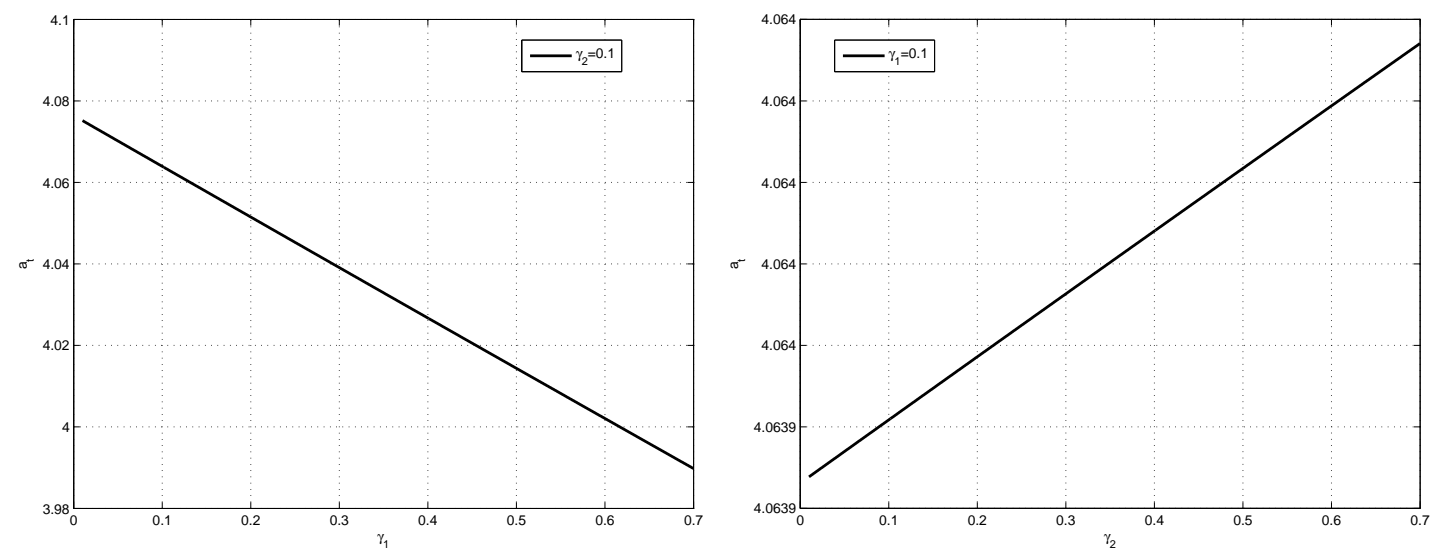

Figure 5: The parameters used in all panels are $\epsilon=0.5, \sigma=0.1, t=0.5, T=1, \xi=0.1$. Left panel: manager's misreporting level versus $\gamma_{1}$ for a fixed level of $\gamma_{2}=0.1$. Right panel: manager's misreporting level versus $\gamma_{2}$ for a fixed level of $\gamma_{1}=0.1$.

$$
\begin{aligned}
\lim _{\epsilon \rightarrow 0} a_{t}^{H A} & =\lim _{\epsilon \rightarrow 0} a_{t}^{R S}=0 \\
\lim _{\gamma_{1} \rightarrow 0} a_{t}^{H A} & =a_{t}^{R S} .
\end{aligned}
$$

Thus, the two actions are also the same and consist in truthful revelation in case $\epsilon \rightarrow 0$, i.e the outcome process $x_{t}=x_{0}$ is constant and known to everyone. In that case there is no beneficial effect of misreporting to manager and shareholders. Moreover, for a very low risk averse manager, the optimal non-contractible misreporting action approaches the joint optimal misreporting action. This is because, as we have seen above, and as it is well known in existing models, when the manager does not care about risk the whole firm is transferred to the manager, $\alpha_{t} \equiv 1$.

It is seen from Fig. 5 that $a_{t}^{H A}$ is decreasing in $\gamma_{1}$. This is expected because a more risk-averse manager is less inclined to misreport due to the penalty incurred. By the same token, since everything is driven by the relative size of risk aversions, the amount of misreporting increases if the shareholders are more risk averse. More precisely, the more risk averse shareholders are, the higher percentage of the firm they give to the manager, who then has incentives to misreport more.

\subsection{Expected utility of Shareholders and Manager}

We compare the expected utilities of shareholders and manager in the risk-sharing and hidden action setting. First, we fix the reservation utility of the manager to $R_{0}$ in both the hidden action and risksharing framework, and measure how the shareholders utility changes. This results in choosing the relative risk-sharing level $\lambda$, so that the manager utility in the risk-sharing case satisfies $J_{1}(T)=R_{0}$. Using Eq. (4.17), we have that this is achieved if

$$
\lambda=\frac{\gamma_{1}}{\gamma_{2}}\left(-\frac{R_{0}}{V}\right)^{1+\frac{\gamma_{2}}{\gamma_{1}}} .
$$



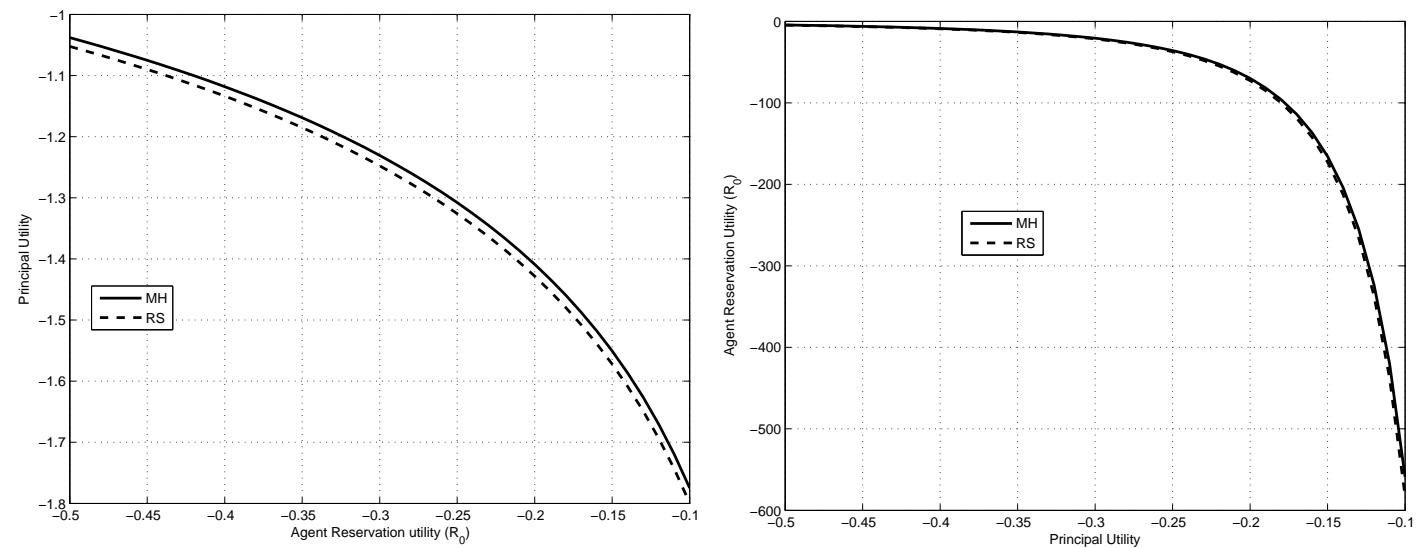

Figure 6: The parameter choices are: $\epsilon=0.2, \sigma=0.1, \theta=0.5, \gamma_{1}=0.3, \gamma_{2}=0.1, x_{0}=1, \xi=0.5$, $T=1$. Left Panel: Shareholders utility under both hidden action (HA) and risk-sharing (RS) as a function of the reservation utility $R_{0}$ of the manager. Right Panel: Manager reservation utility $R_{0}$ under both hidden action (HA) and risk-sharing (RS) as a function of the shareholders utility $x$.

Under this choice of $\lambda$, it is easily seen that the shareholders utility in the risk-sharing framework is given by

$$
J_{2}(T)=-\left(-\frac{R_{0}}{V}\right)^{-\frac{\gamma_{2}}{\gamma_{1}}} V
$$

We have that the shareholders utility in the hidden action setting is given by Eq. (3.13), evaluated using the optimal contract and misreporting action, fully specified by Eq. (3.33) and Eq. (3.34). In particular, for a specified $R_{0}$, we have

$$
\mathbb{E}\left[U_{2}\left(\hat{x}_{T}^{0}-C_{T}\right)\right]=-\left(-R_{0}\right)^{-\frac{\gamma_{2}}{\gamma_{1}}} \exp \left\{-\gamma_{2}\left(x_{0}+\theta \epsilon T\right)+\gamma_{2}\left(d_{1}+d_{3}-K_{1}+K_{3}\right)+\frac{1}{2} \gamma_{2}^{2} K_{2}\right\}
$$

where $d_{1}$ and $d_{3}$ are given by Eq. (3.36) and Eq. (3.38), respectively. We have

$$
K_{1}=\int_{0}^{T} a_{t} \frac{v_{t}}{\sigma^{2}} d t, \quad K_{3}=\int_{0}^{T} \frac{v_{t}}{\sigma} \frac{\epsilon}{\sigma^{2} \cosh \left(\frac{\epsilon t}{\sigma}\right)} p_{t}^{*} d t
$$

with $p_{t}^{*}$ given by Eq. (3.32) and

$$
\begin{aligned}
K_{2} & =\int_{0}^{T}\left(\frac{v_{t}}{\sigma}+\frac{1}{\gamma_{1}} \tilde{Y}_{t}^{a}\right)^{2} d t \\
& =\frac{\gamma_{1}^{2} \operatorname{sech}^{2}(\beta T)\left(4 \beta T \epsilon^{2}-2 \beta^{3} \sigma^{2} T+2 \beta \epsilon^{2} T \cosh (2 \beta T)+\left(\beta^{2} \sigma^{2}-3 \epsilon^{2}\right) \sinh (2 \beta T)\right)}{4 \beta\left(\gamma_{1}+\gamma_{2}\right)^{2}}
\end{aligned}
$$

As expected, the left panel of Fig. 6 shows that the shareholders have a smaller utility when the misreporting level cannot be contracted upon. Moreover, the shareholders utility decreases as the reservation utility of the manager increases.

Next, we fix the shareholders utility to $x$ in both risk-sharing and hidden action framework, and 
measure how the manager utility changes. This requires choosing constant $\lambda$ so that the shareholders utility in the risk-sharing case satisfies $J_{2}(T)=x$. Using Eq. (4.18), we have that this is achieved if

$$
\lambda=\frac{\gamma_{1}}{\gamma_{2}}\left(-\frac{x}{V}\right)^{-\left(1+\frac{\gamma_{1}}{\gamma_{2}}\right)}
$$

Under this choice of $\lambda$, it is easily seen that the manager utility in the risk-sharing framework is given by

$$
J_{1}(T)=-\left(-\frac{x}{V}\right)^{-\frac{\gamma_{1}}{\gamma_{2}}} V
$$

Moreover, if the shareholders utility is $x$, we deduce from Eq. (5.2) that

$$
R_{0}=-\left(-x \exp \left\{\gamma_{2}\left(x_{0}+\theta \epsilon T\right)-\gamma_{2}\left(d_{1}+d_{3}-K_{1}+K_{3}\right)-\frac{1}{2} \gamma_{2}^{2} K_{2}\right\}\right)^{-\frac{\gamma_{1}}{\gamma_{2}}}
$$

which corresponds to the manager utility in the hidden action framework due to Eq. (3.10). Comparing the left and right panel of Fig. 6, we can clearly see that the manager utility is smaller than the shareholders utility due to the manager being more risk averse. Moreover, while for smaller values of shareholders utility the manager's utility is similar in both risk-sharing and hidden action framework, we have that as the shareholders utility gets larger the manager's utility decreases faster if misreporting cannot be contracted upon.

\section{Conclusions}

This paper studied a continuous time model of the firm's value process, where the true outcome can only be observed with noise, and is optimally biased by the manager. More specifically, we consider the situation where both shareholders and manager have unbiased estimates of the actual output process, while the rest of the market is unable to estimate the level of bias introduced by the manager, and therefore does not factor in the correct misreporting level when estimating the process value.

We have shows that the computation of the optimal contract and of the optimal misreporting action applied by the manager can be computed as the solution of a constrained variational calculus problem, for which we provide a closed form solution by means of a methodology that is novel to this type of problems. In order to test the robustness of our conclusions, we considered the risk-sharing case and solved for the general optimal contract. We found it to be linear, with the optimal risksharing fraction determined by the relative size of the risk aversion parameters of the shareholders and the manager. We add to our theoretical results performing a detailed comparative statics analysis. Such analysis reveals that both the optimal misreporting action and pay-per-performance sensitivity increase over time, and that higher levels of observational noise reduce the benefits that both manager and shareholders extract from the market through the misreporting action. As expected, when the cost of misreporting increases, smaller incentives are offered by the shareholders, and smaller levels of misreporting are applied by the manager.

Mathematically, it is an open question whether our results hold true if nonlinear contracts are allowed, or if stochastic action is allowed. Economically, it would be of interest to see how much misreporting would be reduced in the presence of occasional (random) auditing that would reveal existence of misreporting and change the market value of the firm. 


\section{Proofs of Lemmas and Propositions}

\subsection{Proofs for Filtering Estimates}

We prove Lemma 2.1 first.

Proof. Note that Eq. (2.8) gives

$$
d \hat{x}_{s}^{0}=\theta \epsilon d s+\frac{v_{s}}{\sigma} d \hat{Z}_{s}^{0}
$$

which, multiplied with $\frac{\sigma}{v_{s}}$ and integrated from 0 to $t$, gives (2.10). Using Eq. (2.3) and Eq. (2.7), we get

$$
\begin{gathered}
\hat{Z}_{t}^{a}=Z_{t}^{0}-\frac{1}{\sigma} \int_{0}^{t} a_{s} d s+\frac{1}{\sigma} \int_{0}^{t}\left[x_{s}-\hat{x}_{s}^{a}\right] d s \\
\hat{Z}_{t}^{0}=Z_{t}^{0}+\frac{1}{\sigma} \int_{0}^{t}\left[x_{s}-\hat{x}_{s}^{0}\right] d s
\end{gathered}
$$

Subtracting Eq. (7.3) from Eq. (7.2), we arrive at Eq. (2.11). Using (2.10) and (2.11), we obtain Eq. (2.12), which concludes the lemma.

We prove Lemma 2.2 next.

Proof. Let us denote

$$
\omega_{t}=\hat{x}_{t}^{0}-\hat{x}_{t}^{a}
$$

From Eq. (2.11), we have

$$
\frac{d}{d t}\left(\hat{Z}_{t}^{a}-\hat{Z}_{t}^{0}\right)=-\frac{a_{t}}{\sigma}+\frac{\hat{x}_{t}^{0}-\hat{x}_{t}^{a}}{\sigma}
$$

or, equivalently,

$$
\hat{x}_{t}^{0}-\hat{x}_{t}^{a}=\sigma \frac{d}{d t}\left(\hat{Z}_{t}^{a}-\hat{Z}_{t}^{0}\right)+a_{t}
$$

Using Eq. (2.8), we get

$$
\begin{aligned}
\frac{d \hat{x}_{t}^{a}}{d t} & =\theta \epsilon+\frac{v_{t}}{\sigma} \frac{d \hat{Z}_{t}^{a}}{d t} \\
\frac{d \hat{x}_{t}^{0}}{d t} & =\theta \epsilon+\frac{v_{t}}{\sigma} \frac{d \hat{Z}_{t}^{0}}{d t}
\end{aligned}
$$

Using Eq. (7.6) and Eq. (7.7) we obtain

$$
\frac{d}{d t}\left(\hat{x}_{t}^{a}-\hat{x}_{t}^{0}\right)=\frac{v_{t}}{\sigma} \frac{d}{d t}\left(\hat{Z}_{t}^{a}-\hat{Z}_{t}^{0}\right)
$$

and plugging Eq. (7.8) into Eq. (7.5), we obtain

$$
\hat{x}_{t}^{0}-\hat{x}_{t}^{a}=\sigma\left(-\frac{\sigma}{v_{t}} \frac{d}{d t}\left(\hat{x}_{t}^{0}-\hat{x}_{t}^{a}\right)\right)+a_{t}
$$

From the definition of $\omega_{t}$ in Eq. (7.4), and Eq. (7.9), we get the ordinary differential equation

$$
\omega_{t}+\frac{\sigma^{2}}{v_{t}} \omega_{t}^{\prime}=a_{t}
$$


which may be rewritten as

$$
v_{t} \omega_{t}+\sigma^{2} \omega_{t}^{\prime}=a_{t} v_{t}
$$

If we multiply Eq. (7.11) by $\exp \left\{\frac{1}{\sigma^{2}} \int_{0}^{t} v_{s} d s\right\}$, then we have

$$
\exp \left\{\frac{1}{\sigma^{2}} \int_{0}^{t} v_{s} d s\right\} v_{t} \omega_{t}+\sigma^{2} \omega_{t}^{\prime} \exp \left\{\frac{1}{\sigma^{2}} \int_{0}^{t} v_{s} d s\right\}=\exp \left\{\frac{1}{\sigma^{2}} \int_{0}^{t} v_{s} d s\right\} a_{t} v_{t}
$$

which can be rewritten as

$$
\frac{d}{d t}\left(\sigma^{2} \omega_{t} \exp \left\{\frac{1}{\sigma^{2}} \int_{0}^{t} v_{s} d s\right\}\right)=\exp \left\{\frac{1}{\sigma^{2}} \int_{0}^{t} v_{s} d s\right\} a_{t} v_{t}
$$

Using the expression for $v_{t}$ given in Eq. (2.9), we obtain

$$
\frac{1}{\sigma^{2}} \int_{0}^{t} v_{s} d s=\ln \left(\cosh \left(\frac{\epsilon t}{\sigma}\right)\right)
$$

Hence,

$$
\exp \left\{\frac{1}{\sigma^{2}} \int_{0}^{t} v_{s} d s\right\}=\cosh \left(\frac{\epsilon t}{\sigma}\right)
$$

and Eq. (7.13) implies

$$
\frac{d}{d s}\left(\sigma^{2} \omega_{s} \cosh \left(\frac{\epsilon s}{\sigma}\right)\right)=\cosh \left(\frac{\epsilon s}{\sigma}\right) \epsilon \sigma \frac{\sinh \left(\frac{\epsilon s}{\sigma}\right)}{\cosh \left(\frac{\epsilon s}{\sigma}\right)} a_{s}
$$

Integrating Eq. (7.14) from 0 to $t$, we get

$$
\omega_{t}=\frac{\int_{0}^{t} \epsilon \sigma \sinh \left(\frac{\epsilon s}{\sigma}\right) a_{s} d s}{\sigma^{2} \cosh \left(\frac{\epsilon t}{\sigma}\right)}=\frac{\epsilon \int_{0}^{t} \sinh \left(\frac{\epsilon s}{\sigma}\right) a_{s} d s}{\sigma \cosh \left(\frac{\epsilon t}{\sigma}\right)}
$$

which yields the result.

\subsection{Proofs for the Hidden Action Section}

We provide the proof of Lemma 3.3 first.

Proof. For $0<t \leq T$, we have that

$$
p_{t}^{\prime}=\sinh \left(\frac{\epsilon t}{\sigma}\right) a_{t}
$$

and thus, using $a_{t}=\frac{p_{t}^{\prime}}{\sinh \left(\frac{\epsilon t}{\sigma}\right)}$, we can write the integrand as

$$
S\left(t, p_{t}, p_{t}^{\prime}\right) \triangleq \frac{1}{2} \gamma_{1} \alpha_{t}^{2} \frac{v_{t}^{2}}{\sigma^{2}}-\theta \epsilon \alpha_{t}+g\left(\frac{p_{t}^{\prime}}{\sinh \left(\frac{\epsilon t}{\sigma}\right)}\right)-\alpha_{t} \frac{v_{t}}{\sigma^{2}}\left(\frac{p_{t}^{\prime}}{\sinh \left(\frac{\epsilon t}{\sigma}\right)}-\frac{\epsilon}{\cosh \left(\frac{\epsilon t}{\sigma}\right)} \frac{p_{t}}{\sigma}\right) .
$$

Since function $g$ satisfies Eq. (3.1), we have

$$
\lim _{p_{t}^{\prime} \rightarrow \infty} \frac{S\left(t, p_{t}, p_{t}^{\prime}\right)}{p_{t}^{\prime}}=\infty
$$


Moreover, notice that $p_{t}^{\prime} \mapsto S\left(t, p_{t}, p_{t}^{\prime}\right)$ is a convex function. Also, since $\alpha_{t} \geq 0$, we can write

$$
S\left(t, p_{t}, p_{t}^{\prime}\right) \geq h\left(p_{t}^{\prime}\right) \triangleq \frac{1}{2} \gamma_{1} \alpha_{t}^{2} \frac{v_{t}^{2}}{\sigma^{2}}-\theta \epsilon \alpha_{t}+g\left(\frac{p_{t}^{\prime}}{\sinh \left(\frac{\epsilon t}{\sigma}\right)}\right)-\alpha_{t} \frac{v_{t}}{\sigma^{2}}\left(\frac{p_{t}^{\prime}}{\sinh \left(\frac{\epsilon t}{\sigma}\right)}\right)
$$

and see that $S\left(t, p_{t}, p_{t}^{\prime}\right)$ is bounded by function $h\left(p_{t}^{\prime}\right)$, strictly convex in $p_{t}^{\prime}$. Therefore, by Theorem 4.36 in Dacorogna (2007), the function $p_{t}$ yielding the minimum must satisfy the Euler-Lagrange equation given by

$$
\frac{d}{d t}\left[\frac{\partial S}{\partial p_{t}^{\prime}}\right]=\frac{\partial S}{\partial p_{t}}
$$

Using the expression for $S$ in Eq. (7.15), we obtain from Eq. (7.15) that

$$
\frac{d}{d t}\left[g^{\prime}\left(\frac{p_{t}^{\prime}}{\sinh \left(\frac{\epsilon t}{\sigma}\right)}\right) \cdot \frac{1}{\sinh \left(\frac{\epsilon t}{\sigma}\right)}-\alpha_{t} \frac{v_{t}}{\sigma^{2}} \frac{1}{\sinh \left(\frac{\epsilon t}{\sigma}\right)}\right]=\alpha_{t} \frac{v_{t}}{\sigma^{2}} \frac{1}{\sigma} \frac{\epsilon}{\cosh \left(\frac{\epsilon t}{\sigma}\right)}
$$

Moreover, as we have a free boundary at $T$, we have that

$$
\left.\frac{\partial S}{\partial p_{t}^{\prime}}\right|_{t=T}=0
$$

More precisely, we get

$$
g^{\prime}\left(a_{T}\right) \frac{1}{\sinh \left(\frac{\epsilon T}{\sigma}\right)}-\alpha_{T} \frac{v_{T}}{\sigma^{2}} \frac{1}{\sinh \left(\frac{\epsilon T}{\sigma}\right)}=0 .
$$

By substituting $p_{t}^{\prime}=\sinh \left(\frac{\epsilon t}{\sigma}\right) a_{t}$, we therefore rewrite $(7.18)$ as

$$
\frac{d}{d t}\left[g^{\prime}\left(a_{t}\right) \cdot \frac{1}{\sinh \left(\frac{\epsilon t}{\sigma}\right)}-\alpha_{t} \frac{v_{t}}{\sigma^{2}} \frac{1}{\sinh \left(\frac{\epsilon t}{\sigma}\right)}\right]=\alpha_{t} \frac{v_{t}}{\sigma^{2}} \frac{1}{\sigma} \frac{\epsilon}{\cosh \left(\frac{\epsilon t}{\sigma}\right)}
$$

From Eq. (7.19), we also obtain

$$
\alpha_{T} \frac{v_{T}}{\sigma^{2}}=g^{\prime}\left(a_{T}\right)
$$

Rewriting Eq. (7.20) as

$$
\frac{d}{d t}\left[-\alpha_{t} \frac{v_{t}}{\sigma^{2}} \frac{1}{\sinh \left(\frac{\epsilon t}{\sigma}\right)}\right]-\alpha_{t} \frac{v_{t}}{\sigma^{2}} \frac{\epsilon}{\sigma \cosh \left(\frac{\epsilon t}{\sigma}\right)}=-\frac{d}{d t}\left[\frac{g^{\prime}\left(a_{t}\right)}{\sinh \left(\frac{\epsilon t}{\sigma}\right)}\right]
$$

and using the substitution

$$
\Theta_{t}=-\alpha_{t} \frac{v_{t}}{\sigma^{2}} \frac{1}{\sinh \left(\frac{\epsilon t}{\sigma}\right)}
$$

we obtain that (7.22) can be written as

$$
\Theta_{t}^{\prime}+\frac{\epsilon}{\sigma} \tanh \left(\frac{\epsilon t}{\sigma}\right) \Theta_{t}=-\frac{d}{d t}\left[\frac{g^{\prime}\left(a_{t}\right)}{\sinh \left(\frac{\epsilon t}{\sigma}\right)}\right]
$$


Multiplying (7.24) by $\cosh \left(\frac{\epsilon t}{\sigma}\right)$ together with (7.23) gives

$$
\frac{d}{d t}\left[-\alpha_{t} \frac{v_{t}}{\sigma^{2}} \frac{1}{\tanh \left(\frac{\epsilon t}{\sigma}\right)}\right]=-\cosh \left(\frac{\epsilon t}{\sigma}\right) \frac{d}{d t}\left[\frac{g^{\prime}\left(a_{t}\right)}{\sinh \left(\frac{\epsilon t}{\sigma}\right)}\right] .
$$

Integrating (7.25) from $t$ to $T$, and recalling that, by definition, $v_{t}=\epsilon \sigma \tanh \left(\frac{\epsilon t}{\sigma}\right)$, we have

$$
\alpha_{t} \frac{\epsilon}{\sigma}-\alpha_{T} \frac{\epsilon}{\sigma}=-\int_{t}^{T} \cosh \left(\frac{\epsilon s}{\sigma}\right) \frac{d}{d s}\left[\frac{g^{\prime}\left(a_{s}\right)}{\sinh \left(\frac{\epsilon s}{\sigma}\right)}\right] d s .
$$

Using integration by parts on the right hand side together with (7.21) we find the required result in (3.6).

We now provide the proof of Lemma 3.4.

Proof. Note that Eq. (3.1) implies that we can find a convex function $Q\left(p_{t}^{\prime}, q_{t}^{\prime}\right)$ in the form of

$$
Q\left(p_{t}^{\prime}, q_{t}^{\prime}\right)=c_{1}\left(\left(p_{t}^{\prime}\right)^{2}+\left(q_{t}^{\prime}\right)^{2}\right)-\frac{\epsilon}{\sigma} \operatorname{sech}\left(\frac{\epsilon t}{\sigma}\right) p_{t}^{\prime}+c_{2}
$$

for some $c_{1}$ and $c_{2}$ such that

$$
L\left(t, p_{t}, q_{t}, p_{t}^{\prime}, q_{t}^{\prime}\right) \geq Q\left(p_{t}^{\prime}, q_{t}^{\prime}\right)
$$

Hence, the function $\left(p_{t}^{\prime}, q_{t}^{\prime}\right) \mapsto L\left(t, p_{t}, q_{t}, p_{t}^{\prime}, q_{t}^{\prime}\right)$ in (3.19) is superlinear as

$$
\lim _{\left(p_{t}^{\prime}, q_{t}^{\prime}\right) \rightarrow(\infty, \infty)} \frac{L\left(t, p_{t}, q_{t}, p_{t}^{\prime}, q_{t}^{\prime}\right)}{\sqrt{\left(p_{t}^{\prime}\right)^{2}+\left(q_{t}^{\prime}\right)^{2}}}=\infty
$$

and bounded by function $Q$, convex in $\left(p_{t}^{\prime}, q_{t}^{\prime}\right)$. Moreover, a straightforward calculation shows that the map $\left(p_{t}^{\prime}, q_{t}^{\prime}\right) \mapsto L\left(t, p_{t}, q_{t}, p_{t}^{\prime}, q_{t}^{\prime}\right)$ in $(3.19)$ is strictly convex as its Hessian matrix having positive eigenvalues $\xi\left(\operatorname{csch}\left(\frac{\epsilon t}{\sigma}\right)\right)^{2}$ and $\left(\gamma_{1}+\gamma_{2}\right) \sigma^{2}$ is positive definite for $t>0$. Furthermore, the map $\left(p_{t}, q_{t}, p_{t}^{\prime}, q_{t}^{\prime}\right) \mapsto L\left(t, p_{t}, q_{t}, p_{t}^{\prime}, q_{t}^{\prime}\right)$ in (3.19) is convex as its Hessian matrix having nonnegative eigenvalues $0, \xi\left(\operatorname{csch}\left(\frac{\epsilon t}{\sigma}\right)\right)^{2}$ and $\left(\gamma_{1}+\gamma_{2}\right)\left(\sigma^{2}+\epsilon^{2}\left(\tanh \left(\frac{\epsilon t}{\sigma}\right)\right)^{2}\right.$ is positive semi-definite for $t>0$. Application of Theorem 4.36 in Dacorogna (2007) leads to the conclusion that there is unique minimizers $p_{t}$, and $q_{t}$ of $A$ in Eq. (3.14), which together with (3.16), yields the unique minimizer $a_{t}$.

We provide the proof of Lemma 3.5 next.

Proof. Suppose that $\left(p_{t}, q_{t}\right)$ minimizes the functional $\mathcal{J}$ defined by Eq. (3.21). Therefore, any perturbation $\left(p_{t}+\delta_{1} \tilde{p}_{t}, q_{t}+\delta_{2} \tilde{q}_{t}\right)$ of $\left(p_{t}, q_{t}\right)$ would imply

$$
\mathcal{J}\left(p_{t}, q_{t}\right) \leq \mathcal{J}\left(p_{t}+\delta_{1} \tilde{p}_{t}, q_{t}+\delta_{2} \tilde{q}_{t}\right)
$$

where $\delta_{1}$ and $\delta_{2}$ are real numbers and $\tilde{p}_{t}$ and $\tilde{q}_{t}$ are differentiable functions on $[0, T]$ with

$$
\tilde{p}_{0}=0, \quad \tilde{q}_{T}=0 .
$$

Thus, $\left(\delta_{1}, \delta_{2}\right)=(0,0)$ is the critical point of the map $\left(\delta_{1}, \delta_{2}\right) \mapsto \mathcal{J}\left(p_{t}+\delta_{1} \tilde{p}_{t}, q_{t}+\delta_{2} \tilde{q}_{t}\right)$. Therefore, we have

$$
\left.\frac{\partial}{\partial \delta_{1}} \mathcal{J}\left(p_{t}+\delta_{1} \tilde{p}_{t}, q_{t}+\delta_{2} \tilde{q}_{t}\right)\right|_{\left(\delta_{1}, \delta_{2}\right)=(0,0)}=0
$$


and

$$
\left.\frac{\partial}{\partial \delta_{2}} \mathcal{J}\left(p_{t}+\delta_{1} \tilde{p}_{t}, q_{t}+\delta_{2} \tilde{q}_{t}\right)\right|_{\left(\delta_{1}, \delta_{2}\right)=(0,0)}=0
$$

By (7.28) we have

$$
\int_{0}^{T}\left(\frac{\partial L}{\partial p_{t}} \tilde{p}_{t}+\frac{\partial L}{\partial p_{t}^{\prime}} \tilde{p}_{t}^{\prime}+\lambda(t)\left[g^{\prime \prime}\left(\operatorname{csch}\left(\frac{\epsilon t}{\sigma}\right) p_{t}^{\prime}\right) \operatorname{csch}\left(\frac{\epsilon t}{\sigma}\right) \tilde{p}_{t}^{\prime}\right]\right) d t=0
$$

Application of integration by parts and using the free boundary $\alpha_{T}$ give Equations (3.23) and (3.25). Also, by (7.29) we have

$$
\int_{0}^{T}\left(\frac{\partial L}{\partial q_{t}} \tilde{q}_{t}+\frac{\partial L}{\partial q_{t}^{\prime}} \tilde{q}_{t}^{\prime}+\lambda(t) \tilde{q}_{t}^{\prime}\right) d t=0 .
$$

Again using integration by parts and the free boundary $\beta_{0}$ results in Equations (3.24) and (3.26).

Next, we provide the proof of Proposition 3.2.

Proof. We solve the system of Equations (3.23)-(3.27) in Lemma 3.5. To ease the notation we drop the superscript $H A$ in the proof. First, we differentiate $L$ with respect to $p_{t}$ and $p_{t}^{\prime}$, and obtain

$$
\frac{\partial L}{\partial p_{t}}=\frac{\epsilon^{2}}{\sigma^{2}} \tanh \left(\frac{\epsilon t}{\sigma}\right) \operatorname{sech}\left(\frac{\epsilon t}{\sigma}\right)=-\frac{d}{d t}\left(\frac{\epsilon}{\sigma} \operatorname{sech}\left(\frac{\epsilon t}{\sigma}\right)\right)
$$

and

$$
\frac{\partial L}{\partial p_{t}^{\prime}}=\xi\left(\operatorname{csch}\left(\frac{\epsilon t}{\sigma}\right)\right)^{2} p_{t}^{\prime}-\frac{\epsilon}{\sigma} \operatorname{sech}\left(\frac{\epsilon t}{\sigma}\right)
$$

Note that by Eq. (3.29) we have

$$
p_{T}^{\prime}=-\frac{1}{\xi} \sinh \left(\frac{\epsilon T}{\sigma}\right) q_{T}^{\prime}
$$

Moreover, Eq. (3.25) simplifies to

$$
\xi\left(\operatorname{csch}\left(\frac{\epsilon T}{\sigma}\right)\right)^{2} p_{T}^{\prime}-\frac{\epsilon}{\sigma} \operatorname{sech}\left(\frac{\epsilon T}{\sigma}\right)=-\xi \lambda(T) \operatorname{csch}\left(\frac{\epsilon T}{\sigma}\right)
$$

or, by using Eq. (7.34),

$$
\lambda(T)=\frac{1}{\xi} q_{T}^{\prime}+\frac{\epsilon}{\sigma \xi} \tanh \left(\frac{\epsilon T}{\sigma}\right) .
$$

Also, Eq. (3.23) is equivalent to

$$
\frac{d}{d t}\left[\left(\operatorname{csch}\left(\frac{\epsilon t}{\sigma}\right)\right)^{2} p_{t}^{\prime}+\operatorname{csch}\left(\frac{\epsilon t}{\sigma}\right) \lambda(t)\right]=0
$$

which by Eq. (3.29) can be written as

$$
\frac{d}{d s}\left[\left(\lambda(s)-\frac{1}{\xi} q_{s}^{\prime}\right) \operatorname{csch}\left(\frac{\epsilon s}{\sigma}\right)\right]=0 .
$$


Integrating Eq. (7.38) from $t$ to $\mathrm{T}$ and using (7.36) we get

$$
\lambda(t)=\frac{1}{\xi} q_{t}^{\prime}+\frac{\epsilon}{\sigma \xi} \operatorname{sech}\left(\frac{\epsilon T}{\sigma}\right) \sinh \left(\frac{\epsilon t}{\sigma}\right) .
$$

which yields

$$
\lambda^{\prime}(t)=\frac{1}{\xi} q_{t}^{\prime \prime}+\frac{\epsilon^{2}}{\sigma^{2} \xi} \operatorname{sech}\left(\frac{\epsilon T}{\sigma}\right) \cosh \left(\frac{\epsilon t}{\sigma}\right) .
$$

We next use Eq. (3.24) to obtain another expression for $\lambda^{\prime}(t)$. Differentiating $L$ with respect to $q_{t}$ and $q_{t}^{\prime}$, we obtain

$$
\frac{\partial L}{\partial q_{t}}=\left(\gamma_{1}\left[\sigma q_{t}^{\prime}-\epsilon \tanh \left(\frac{\epsilon t}{\sigma}\right) q_{t}\right]+\gamma_{2}\left[\left(1-q_{t}\right) \epsilon \tanh \left(\frac{\epsilon t}{\sigma}\right)+\sigma q_{t}^{\prime}\right]\right)\left(-\epsilon \tanh \left(\frac{\epsilon t}{\sigma}\right)\right)
$$

and

$$
\frac{\partial L}{\partial q_{t}^{\prime}}=\sigma \gamma_{1}\left(\sigma q_{t}^{\prime}-\epsilon \tanh \left(\frac{\epsilon t}{\sigma}\right) q_{t}\right)+\sigma \gamma_{2}\left(\left(1-q_{t}\right) \epsilon \tanh \left(\frac{\epsilon t}{\sigma}\right)+\sigma q_{t}^{\prime}\right)
$$

Due to Eq. (3.26) we have

$$
\lambda(0)=-\left.\frac{\partial L}{\partial q_{t}^{\prime}}\right|_{t=0}=-\sigma^{2}\left(\gamma_{1}+\gamma_{2}\right) q_{0}^{\prime}
$$

which combined with $\lambda(0)=\frac{1}{\xi} q_{0}^{\prime}$ from (7.39), implies

$$
q_{0}^{\prime}=0
$$

Furthermore, it is not difficult to compute that

$$
\frac{d}{d t} \frac{\partial L}{\partial q_{t}^{\prime}}=\left(\gamma_{1}+\gamma_{2}\right) \sigma^{2} q_{t}^{\prime \prime}-\left(\gamma_{1}+\gamma_{2}\right) \epsilon \sigma q_{t}^{\prime} \tanh \left(\frac{\epsilon t}{\sigma}\right)+\epsilon^{2} \operatorname{sech}\left(\frac{\epsilon t}{\sigma}\right)^{2}\left(\gamma_{2}\left(1-q_{t}\right)-\gamma_{1} q_{t}\right)
$$

Using Eq. (3.24) together with Eq. (7.41) and Eq. (7.45) we arrive at

$$
\begin{aligned}
\lambda^{\prime}(t) & =\frac{\partial L}{\partial q_{t}}-\frac{d}{d t}\left(\frac{\partial L}{\partial q_{t}^{\prime}}\right) \\
& =-\left(\gamma_{1}+\gamma_{2}\right) \sigma^{2} q_{t}^{\prime \prime}+\left(\gamma_{1}+\gamma_{2}\right) \epsilon^{2} q_{t}-\gamma_{2} \epsilon^{2}
\end{aligned}
$$

Comparing Eq. (7.40) with Eq. (7.46), we conclude that the following second order differential equation has to hold

$$
-\left(\left(\gamma_{1}+\gamma_{2}\right) \sigma^{2}+\frac{1}{\xi}\right) q_{t}^{\prime \prime}+\left(\gamma_{1}+\gamma_{2}\right) \epsilon^{2} q_{t}-\frac{\epsilon^{2}}{\sigma^{2} \xi} \operatorname{sech}\left(\frac{\epsilon T}{\sigma}\right) \cosh \left(\frac{\epsilon t}{\sigma}\right)-\gamma_{2} \epsilon^{2}=0
$$

which is equivalent to the following form

$$
q_{t}^{\prime \prime}-\beta^{2} q_{t}+\varrho \cosh \left(\frac{\epsilon t}{\sigma}\right)+\tau=0
$$


where $\beta, \varrho$, and $\tau$ are given by

$$
\beta=\left(\frac{\epsilon^{2} \xi\left(\gamma_{1}+\gamma_{2}\right)}{\left(\gamma_{1}+\gamma_{2}\right) \sigma^{2} \xi+1}\right)^{1 / 2}, \quad \varrho=\frac{\epsilon^{2} \operatorname{sech}\left(\frac{\epsilon T}{\sigma}\right)}{\left(\left(\gamma_{1}+\gamma_{2}\right) \sigma^{2} \xi+1\right) \sigma^{2}}, \quad \tau=\frac{\gamma_{2} \epsilon^{2} \xi}{\left(\gamma_{1}+\gamma_{2}\right) \sigma^{2} \xi+1}
$$

The solution $q_{t}$ of $(7.48)$ can be written as

$$
\begin{aligned}
q_{t} & =c_{1} e^{\beta t}+c_{2} e^{-\beta t}+\frac{\tau\left(\beta^{2} \sigma^{2}-\epsilon^{2}\right)+\beta^{2} \varrho \sigma^{2} \cosh \left(\frac{\epsilon t}{\sigma}\right)}{\beta^{2}\left(\beta^{2} \sigma^{2}-\epsilon^{2}\right)} \\
& =c_{1} e^{\beta t}+c_{2} e^{-\beta t}-\operatorname{sech}\left(\frac{\epsilon T}{\sigma}\right) \cosh \left(\frac{\epsilon t}{\sigma}\right)+\frac{\gamma_{2}}{\gamma_{1}+\gamma_{2}} .
\end{aligned}
$$

as by (7.49) we have

$$
\frac{\tau}{\beta^{2}}=\frac{\gamma_{2}}{\gamma_{1}+\gamma_{2}}, \quad \frac{\varrho \sigma^{2}}{\beta^{2} \sigma^{2}-\epsilon^{2}}=-\operatorname{sech}\left(\frac{\epsilon T}{\sigma}\right)
$$

Eq. (7.48) may be solved explicitly by using the conditions $q_{T}=0$ and $q_{0}^{\prime}=0$, yielding

$$
c_{1}=c_{2}=\frac{\gamma_{1}}{2\left(\gamma_{1}+\gamma_{2}\right)} \operatorname{sech}(\beta T)
$$

As a result, we deduce that

$$
q_{t}=\frac{\gamma_{1}}{\gamma_{1}+\gamma_{2}} \operatorname{sech}(\beta T) \cosh (\beta t)-\operatorname{sech}\left(\frac{\epsilon T}{\sigma}\right) \cosh \left(\frac{\epsilon t}{\sigma}\right)+\frac{\gamma_{2}}{\gamma_{1}+\gamma_{2}}
$$

It follows from Eq. (7.51) that

$$
q_{t}^{\prime}=\frac{\gamma_{1} \beta}{\gamma_{1}+\gamma_{2}} \operatorname{sech}(\beta T) \sinh (\beta t)-\frac{\epsilon}{\sigma} \operatorname{sech}\left(\frac{\epsilon T}{\sigma}\right) \sinh \left(\frac{\epsilon t}{\sigma}\right) .
$$

Also, we know that

$$
\begin{aligned}
p_{t}= & -\frac{1}{\xi} \int_{0}^{t} \sinh \left(\frac{\epsilon s}{\sigma}\right) q_{s}^{\prime} d s \\
= & \frac{\beta \gamma_{1} \sigma \operatorname{sech}(\beta T)\left(-\epsilon \cosh \left(\frac{\epsilon t}{\sigma}\right) \sinh (\beta t)+\beta \sigma \cosh (\beta t) \sinh \left(\frac{\epsilon t}{\sigma}\right)\right)}{\left(\gamma_{1}+\gamma_{2}\right)\left(\epsilon^{2}-\beta^{2} \sigma^{2}\right) \xi} \\
& +\frac{\operatorname{sech}\left(\frac{\epsilon T}{\sigma}\right)\left(\sigma \sinh \left(\frac{2 \epsilon t}{\sigma}\right)-2 \epsilon t\right)}{4 \xi \sigma} .
\end{aligned}
$$

\subsection{Proofs for Risk Sharing Case}

We provide a proof for Lemma 4.6

Proof. Notice that $p_{t}^{\prime} \mapsto \tilde{L}\left(t, p_{t}, p_{t}^{\prime}\right)$ is strictly convex. Moreover, we have

$$
\lim _{p_{t}^{\prime} \rightarrow \infty} \frac{\tilde{L}\left(t, p_{t}, p_{t}^{\prime}\right)}{p_{t}^{\prime}}=\infty
$$


and

$$
\tilde{L}\left(t, p_{t}, p_{t}^{\prime}\right) \geq m\left(p_{t}^{\prime}\right) \triangleq-\theta \epsilon-\frac{\epsilon}{\sigma} \operatorname{sech}\left(\frac{\epsilon t}{\sigma}\right) p_{t}^{\prime}+\frac{\xi}{2}\left(\operatorname{csch}\left(\frac{\epsilon t}{\sigma}\right)\right)^{2}\left(p_{t}^{\prime}\right)^{2}+\frac{1}{2} \epsilon^{2} \Gamma\left(\tanh \left(\frac{\epsilon t}{\sigma}\right)\right)^{2}
$$

where $m\left(p_{t}^{\prime}\right)$ is a convex function of $p_{t}^{\prime}$. Therefore, there is a function $p_{t}$ minimizing $\tilde{I}$ by Theorem 4.36 in Dacorogna (2007). Thus, we get $a_{t}$ by(4.11).

We now provide a proof for Lemma 4.7.

Proof. Observe that

$$
\frac{\partial \tilde{L}}{\partial p_{t}}=\frac{\epsilon^{2}}{\sigma^{2}} \tanh \left(\frac{\epsilon t}{\sigma}\right) \operatorname{sech}\left(\frac{\epsilon t}{\sigma}\right), \quad \frac{\partial \tilde{L}}{\partial p_{t}^{\prime}}=\xi\left(\operatorname{csch}\left(\frac{\epsilon t}{\sigma}\right)\right)^{2} p_{t}^{\prime}-\frac{\epsilon}{\sigma} \operatorname{sech}\left(\frac{\epsilon t}{\sigma}\right) .
$$

Since $p_{T}$ is free, we have

$$
\left.\frac{\partial \tilde{L}}{\partial p_{t}^{\prime}}\right|_{t=T}=0
$$

Integrating

$$
\frac{d}{d t} \frac{\partial \tilde{L}}{\partial p_{t}^{\prime}}=\frac{\partial \tilde{L}}{\partial p_{t}}
$$

from $t$ to $T$ and taking into account (7.54) we obtain

$$
p_{t}^{\prime}=\frac{\epsilon}{\xi \sigma} \frac{\left(\sinh \left(\frac{\epsilon t}{\sigma}\right)\right)^{2}}{\cosh \left(\frac{\epsilon T}{\sigma}\right)} .
$$

Integrating Eq. (7.55) from 0 to $t$ and recalling $p_{0}=0$ we obtain Eq. (4.14).

\subsection{Proofs for Comparative Statics}

We prove that $\alpha_{t}^{H A}$ is a decreasing function of $\epsilon$. We may rewrite it as

$$
\alpha_{t}^{H A}=\frac{\gamma_{2}}{\gamma_{1}+\gamma_{2}} \frac{\cosh (\epsilon \zeta t)}{\cosh (\epsilon \zeta T)}\left[1-\zeta \sigma \frac{\tanh (\epsilon \zeta t)}{\tanh \left(\frac{\epsilon t}{\sigma}\right)}\right]
$$

where

$$
\zeta \triangleq \frac{\sqrt{\left(\gamma_{1}+\gamma_{2}\right) \xi}}{\sqrt{\left(\gamma_{1}+\gamma_{2}\right) \sigma^{2} \xi+1}}<\frac{1}{\sigma}
$$

We first show that $\frac{\cosh (\epsilon \zeta t)}{\cosh (\epsilon \zeta T)}$ is a decreasing function of $\epsilon$. Therefore, we compute

$$
\begin{aligned}
\frac{d \frac{\cosh (\epsilon \zeta t)}{d \epsilon} \frac{\cosh (\epsilon \zeta T)}{}}{} & \operatorname{sech}(\epsilon \zeta T)[\zeta t \sinh (\epsilon \zeta t)-\zeta t \cosh (\epsilon \zeta t) \tanh (\epsilon \zeta T)] \\
& \leq \operatorname{sech}(\epsilon \zeta T)[\zeta(t-T) \sinh (\epsilon \zeta t)] \\
& \leq 0
\end{aligned}
$$


for $t \leq T$. Note that since $0<\zeta<1 / \sigma$ we get

$$
0<r(\epsilon) \triangleq 1-\zeta \sigma \frac{\tanh (\epsilon \zeta t)}{\tanh \left(\frac{\epsilon t}{\sigma}\right)} \leq 1
$$

We next show that $r(\epsilon)$ is a decreasing function of $\epsilon$. This follows from the fact that if $t_{1}<t_{2}$ we have

$$
\frac{d}{d \epsilon} \frac{\tanh \left(\epsilon t_{1}\right)}{\tanh \left(\epsilon t_{2}\right)}=t_{1} \operatorname{coth}\left(\epsilon t_{2}\right) \operatorname{sech}^{2}\left(\epsilon t_{1}\right)-t_{2} \operatorname{csch}^{2}\left(\epsilon t_{2}\right) \tanh \left(\epsilon t_{1}\right)>0
$$

which may be seen by simply showing that

$$
\frac{\sinh \left(2 \epsilon t_{2}\right)}{2 t_{2}}>\frac{\sinh \left(2 \epsilon t_{1}\right)}{2 t_{1}}
$$

Consequently, $\alpha_{t}^{H A}$ being the product of two positive decreasing functions decreases in $\epsilon$.

\section{References}

Capponi, A., and Cvitanić, J. (2008): Credit Risk Modeling with Misreporting and Incomplete Information. International J. of Theoretical and Applied Finance 12 81-112.

Carlier, G., Ekeland, I., and Touzi, N. (2007): Optimal derivatives design for mean-variance agents under adverse selection. Mathematics and Financial Economics 1 57-80.

Cox, A.M., and Hobson, D.G. (2005): Local martingales, bubbles and option prices. Finance and Stochastics 9 477-492.

Cuoco, D. and Kaniel, R. (2011): Equilibrium prices in the presence of delegated portfolio management. Journal of Financial Economics 101 264-296.

Cvitanić, J., Wan, X., and Zhang, J. (2009): Optimal Compensation with Hidden Action and LumpSum Payment in a Continuous-Time Model. Applied Mathematics and Optimization 59 99-146.

Dacorogna, B. (2007): Direct Methods in the Calculus of Variation, 2nd edition. Applied Mathematical Sciences, Springer.

Figalli, A., Kim, Y.H., and McCann, R.J. (2011): When is multidimensional screening a convex program? J. Econom. Theory 146, 454-478.

Fishman, M., Hagerty, K. 2003. Mandatory vs voluntary disclosure in markets with informed and uninformed customers. Journal of Law, Economics and Organization 19, 45-63.

Giat, Y., Hackman, S.T., and Subramanian, A. (2010): Investment under Uncertainty, Heterogeneous Beliefs and Agency Conflicts. The Review of Financial Studies 23 1360-1404.

Goukasian, L. and Wan, X (2010). Optimal Incentive Contracts under Relative Income Concerns. Mathematics and Financial Economics 4 57-86.

Holmstrom, B., and Milgrom, P. (1987): Aggregation and Linearity in the Provision of Intertemporal Incentives. Econometrica 55 303-328. 
Jarrow, R.A., Protter, P., and Shimbo, K. (2010): Asset price bubbles in incomplete markets. Mathematical Finance 20 145-185.

Ju, N. and Wan, X. (2010): Optimal compensation and pay-performance sensitivity in a continuoustime principal-agent model. Working paper, HKUST.

Horst, U., Moreno-Bromberg, S. (2008): Risk minimization and optimal derivative design in a principal agent game. Mathematics and Financial Economics 2 1-27.

Liptser, R.S., Shiryaev, A.N. (2000): Statistics of Random Processes II. Applications, SpringerVerlag, New York.

Loewenstein, M., and Willard, G.A. (2000): Rational equilibrium asset-pricing bubbles in continuous trading models. Journal of Economic Theory 91 17-58.

Ou-Yang, H. (2005): An equilibrium model of asset pricing and moral hazard. Review of Financial Studies 18 1253-1303.

Schättler, H., and Sung, J. (1993): The First-Order Approach to Continuous-Time Principal-Agent Problem with Exponential Utility. Journal of Economic Theory 61 331-371.

Schättler, H., J. Sung. (1997): On Optimal Sharing Rules in Discrete and Continuous-Times Principal-Agent Problems with Exponential Utility. Journal of Economic Dynamics and Control 21 551-574. 